\title{
Strength-frequency curve for micromagnetic neurostimulation through EPSPs on rat hippocampal neurons and numerical modeling of magnetic microcoil ( $\mu$ coil)
}

Renata Saha ${ }^{1}$, Sadegh Faramarzi ${ }^{2}$, Robert P. Bloom ${ }^{1}$, Onri J. Benally ${ }^{1}, \mathrm{Kai} \mathrm{Wu}^{1}$, Arturo di Girolamo ${ }^{1}$, Denis Tonini ${ }^{1}$, Susan A. Keirstead ${ }^{3}$, Walter C. Low ${ }^{4}$, Theoden I. Netoff ${ }^{2}$, Jian-Ping Wang ${ }^{1 *}$

${ }^{1}$ Department of Electrical and Computer Engineering, University of Minnesota, Minneapolis, MN, United

States

${ }^{2}$ Department of Biomedical Engineering, University of Minnesota, Minneapolis, MN, United States

${ }^{3}$ Department of Integrative Biology \& Physiology, University of Minnesota, Minneapolis, MN, United

States

${ }^{4}$ Department of Neurosurgery, University of Minnesota, Minneapolis, MN, United States

*Corresponding author: jpwang@umn.edu

\begin{abstract}
Objective: The objective of this study was to measure the effect of micromagnetic stimulation ( $\mu \mathrm{MS}$ ) on hippocampal neurons, by using single microcoil ( $\mu$ coil) prototype, Magnetic Pen (MagPen). MagPen will be used to stimulate the CA3 region magnetically and excitatory post synaptic potential (EPSP) response measurements will be made from the CA1 region. The threshold for micromagnetic neurostimulation as a function of stimulation frequency of the current driving the $\mu$ coil will be demonstrated. Finally, the optimal stimulation frequency of the current driving the $\mu$ coil to minimize power will be estimated. Approach: A biocompatible, watertight, non-corrosive prototype, MagPen was built, and customized such that it is easy to adjust the orientation of the $\mu$ coil and its distance over the hippocampal tissue in an in vitro recording setting. Finite element modeling (FEM) of the $\mu$ coil design was performed to estimate the spatial profiles of the magnetic flux density (in T) and the induced electric fields (in $\mathrm{V} / \mathrm{m}$ ). The induced electric field profiles generated at different values of current applied to the $\mu$ coil can elicit a neuron response, which was validated by numerical modeling. The modeling settings for the $\mu$ coil were replicated in experiments on rat hippocampal neurons. Main results: The preferred orientation of MagPen over the Schaffer Collateral fibers was demonstrated such that they elicit a neuron response. The recorded EPSPs from CA1 region due to $\mu \mathrm{MS}$ at CA3 region were validated by applying tetrodotoxin (TTX). Application of TTX to the hippocampal slice blocked the EPSPs from $\mu \mathrm{MS}$ while after prolonged TTX washout, a partial recovery of the EPSP from $\mu \mathrm{MS}$ was observed. Finally, it was interpreted through numerical analysis that increasing frequency of the current driving the $\mu$ coil, led to a decrease in the current amplitude threshold for micromagnetic neurostimulation. Significance: This work reports that micromagnetic neurostimulation can be used to evoke population EPSP responses in the CA1 region of the hippocampus. It demonstrates the strengthfrequency curve for $\mu \mathrm{MS}$ and its unique features related to orientation dependence of the $\mu$ coils, spatial selectivity and stimulation threshold related to distance dependence. Finally, the challenges related to $\mu \mathrm{MS}$ experiments were studied including ways to overcome them.
\end{abstract}

\section{Introduction}

The market of neurostimulation devices and systems is estimated to reach $\$ 11$ billion per year by 2026 with a compound annual growth rate (CAGR) of $12.5 \%$ [1-3]. In terms of device type, spinal cord stimulators [4-6] and deep brain stimulators [7-9] are expected lead the market, with applications to chronic pain management [10-12] and hearing loss treatments [13-15]. With the increasing rate of FDA-approved treatment options for epilepsy [16], dystonia [8], Tourette's syndrome [7], Parkinson's Disease [17], chronic pain [10] etc. using electrical electrodes, neuromodulation devices have a bright future. However, the existing electrical implants have their own drawbacks. They have a uniform spread of activation and must be in galvanic contact with the biological tissues. After years of implantation, due to inflammatory reactions from the neighboring tissues, the efficacy of electrical stimulation may abate due to biofouling of the electrodes [18-20]. If encapsulation occurs, electrodes may need to be replaced through a revision surgery [21,22]. While many electrodes are approved for magnetic resonance imaging (MRI), for safety concerns they are limited to low field and low resolution devices [23]. On the contrary, transcranial magnetic stimulation (TMS) is a non-invasive therapy which has been FDA-approved for depression [24] 
and obsessive-compulsive disorder (OCD) [25]. It uses a time-varying magnetic field that can permeate deep through the skull to stimulate the brain. This time-varying magnetic field induces an electric field in neural tissue to modulate firing rate of neurons. While there are other neuromodulation therapies, such as chemical [26], optical [27], and ultrasound [28], the neuromodulatory device presented in this work will be compared to implantable electrical electrodes and TMS therapy.

When electrical stimulation is applied through an electrode implanted on the surface, or deep in the brain, it produces very focal volumes of activation. Magnetic stimulation is applied transcranially, and while it is non-invasive it does not have the focality of implanted electrodes. The motivation for developing this device was to make a $\mu \mathrm{MS}$ device with the focality of an implantable electrode. Implantable $\mu \mathrm{MS}$ has been demonstrated previously by Bonmassar et al.[29] in 2012 using commercially available sub-mm sized $\mu$ coils to stimulate rabbit retinal neurons. By applying a time-varying current through a $\mu$ coil, as per Faraday's law of electromagnetic induction, a time-varying magnetic field is generated. This magnetic field then induces an electric field in the tissue surrounding the coil, which is spatially asymmetric. The electric filed gradients generated by the $\mu$ coil may activate small, discrete populations at predictable locations relative to the coil.

Micromagnetic neurostimulation has advantage over electrical stimulation in that it doesn't require an electrochemical interface. This allows flexibility in applying many waveforms to drive these magnetic $\mu$ coils. The application of a wide range of waveforms to drive electrical electrodes in neurostimulation are limited [30,31]. Unbalanced waveforms cause corrosion of the electrodes through irreversible redox reactions at the tissue-electrode interface. Multiple research groups have reported the fabrication of customized $\mu$ coils [32,33], of different shapes and dimensions, including planar [34,35], trapezoidal [32] or solenoidal $[29,36]$ or V-shaped bent wires [32]. These $\mu$ coil designs have been shown to modulate different populations of neurons, in both in vitro and in vivo settings: L5 pyramidal neurons [37], intracortical neurons [32] and inferior colliculus (IC) [36] neurons. In addition, recent numerical studies have shown that these $\mu$ coils are MRI compatible [38] as they show very little to no heating around the implant area in an MRI environment. This is because there is no galvanic contact with adjacent tissues, unlike the electrical implants, thereby limiting the amount of heat generation. However, the power of operation of these $\mu$ coils is three orders of magnitude higher than DBS leads [29]. Recently, to combat this, spintronic nanodevices [39-41] having ultra-low power of operation has been theoretically proved to have therapeutic neuromodulation capability through implantable magnetic stimulation.

In this work, we have tested $\mu$ coil in rat hippocampal slice and constructed the strength-frequency curve for micromagnetic neurostimulation forecasting low power operation of these micromagnetic implants at higher frequency. To validate the EPSPs responses, we have blocked and partially reversed the response with TTX. We will also discuss the unique features of micromagnetic neurostimulation related to spatial selectivity, distance dependence and orientation dependence.

\section{Material and methods}

\subsection{The implantable micromagnetic neurostimulation prototype}

The prototype of the micromagnetic neurostimulation implant, MagPen, investigated in this work is shown in Fig. 1(a). Commercially available $\mu$ coils (Panasonic ELJ-RFR10JFB) were soldered (using solder flux and a hot air blower) at the tip of a $3 \mathrm{~cm}$ long pen-shaped printed circuit board (PCB). The thickness of the PCB board along the z-direction was thinned down to $0.4 \mathrm{~mm}$ to facilitate easier adjustment of the MagPen prototype over the hippocampal slices during in vitro experiments. The preferred orientation of the $\mu$ coil that would effectively stimulate neurons was unknown. Hence two different orientations of the MagPen were designed: (1) Type horizontal or Type $\mathrm{H}$ and (2) Type vertical or Type V. The pen-shaped tip of the Type $\mathrm{H}$ and Type $\mathrm{V}$ prototypes is $1.7 \mathrm{~mm}$ and $1.4 \mathrm{~mm}$, respectively. The complete prototype is shown in Fig. S2(a) of Supplementary Information S2.

Both prototypes were encapsulated by $2 \mu \mathrm{m}$ thick Parylene-C coating using the SCS LabCoater Parylene-C deposition system. The coating was used to make the MagPen prototype biocompatible, noncorrosive, and to insulate the electronics to prevent any possible neurostimulation through leakage current and capacitive coupling from these $\mu$ coils. Successful watertight and anti-leakage current coating for 
MagPen was ensured by measuring the impedance of one terminal of MagPen (see Fig. S2(a)) to an external electrode in artificial cerebrospinal fluid (aCSF) solution (see section 2.6). If that impedance measured above $5 \mathrm{M} \Omega$, that prototype was considered to be a successful prototype for micromagnetic neurostimulation study. In the design of the micromagnetic stimulation implants, this watertight and biocompatible coating is of utmost importance to assure that any neuronal response was caused by the induced electric field only. Fig. 1(b) compares the sub-mm size of the $\mu$ coil to that of a single rice grain.

\subsection{The electrical circuit equivalence of the $\mu$ coil}

Resistance (R), inductance (L) and capacitance (C) measurements of the $\mu$ coil at $1 \mathrm{kHz}$ using an LCR meter (Model no. BK Precision 889B; see Supplementary Information S1) showed that the electrical circuit equivalent of the $\mu$ coil within this frequency range is a series RL circuit (see Fig. 1(c)). Following from laws of electromagnetic induction, the inductance $\left(\mathrm{L}_{\mathrm{s}}\right)$ is directly proportional to the electromotive force (emf), $v(t)=L \frac{d i}{d t}$, where $v(t)$ is the emf induced in an electrical circuit (here, neurons) due to a timevarying magnetic flux density; $v(t)$ contributes directly to the induced electric field which is used to stimulate the neurons and $\frac{d i}{d t}$ is the time-varying current through the inductor or $\mu$ coil. The resistance $\left(\mathrm{R}_{\mathrm{DC}}\right)$ contributes to heat dissipation from the electrical circuit. Future designs of the micromagnetic neurostimulation implants will be focused on further reducing the resistance because lower resistance coils will generate less heat and for the same magnetic field.

\subsection{The $\mu$ coil driving circuitry}

To test the $\mu$ coils in brain slice, they were powered by 1-cycle of sinusoidal current of amplitudes 2 A, frequency $2 \mathrm{kHz}$, phase $0^{\circ}$ and offset at $0 \mathrm{mV}$. Each cycle of pulse is separated by a duration of 5 secs (see Fig. 1(c)). The $5 \mathrm{sec}$ pause between the stimulation pulses was to assure synaptic plasticity effects were not induced in the synaptic coupling between the hippocampal neurons [42,43]. The current waveform was generated using a function generator (model no. Tektronix AFG2021) and amplified using a MOSFET class-D amplifier of $70 \mathrm{kHz}$ bandwidth (model no. Pyramid PB717X). Fig. 2(d) - left shows the signal flow chain to power the $\mu$ coils for EPSP recording studies in this work. 

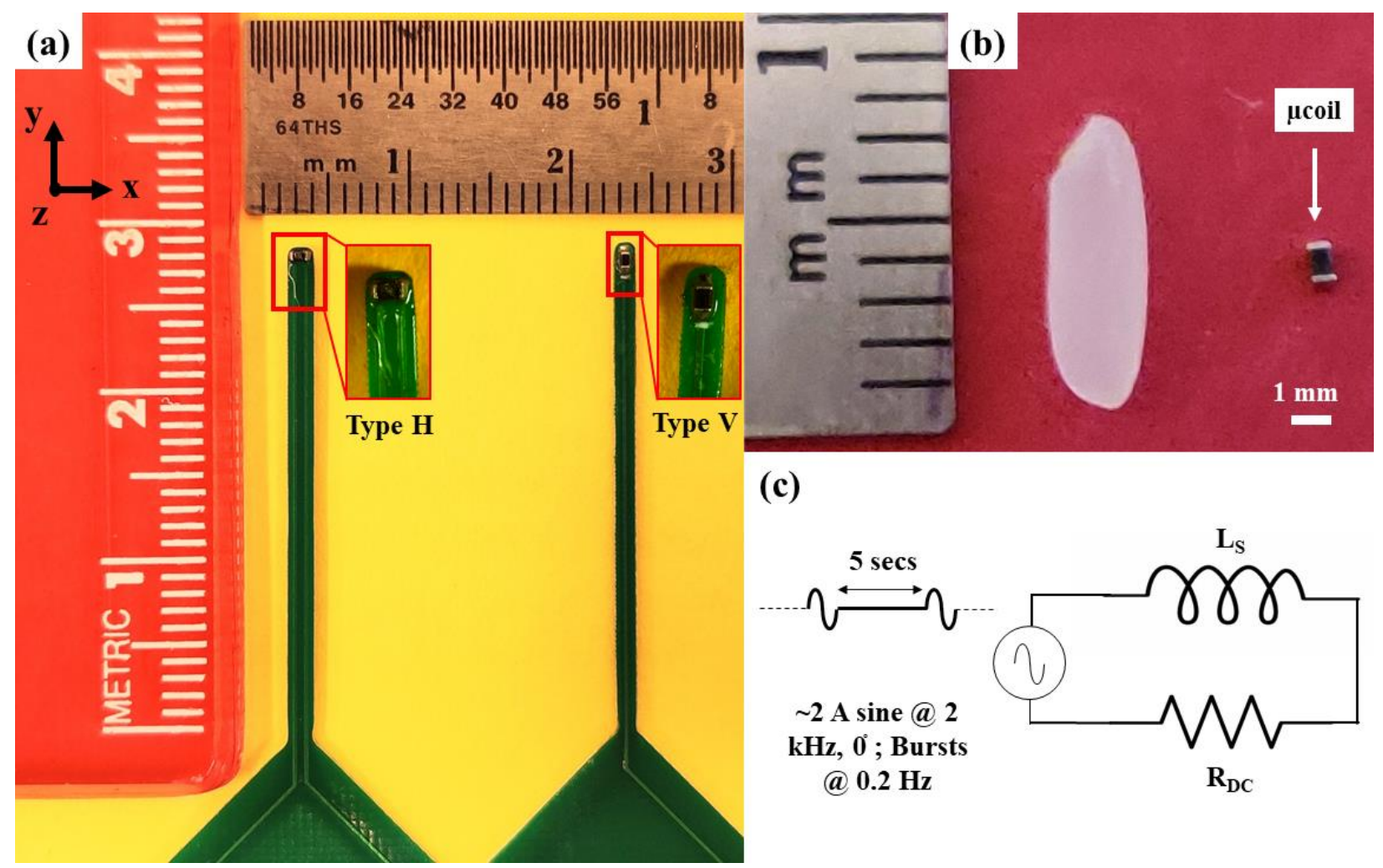

(c)

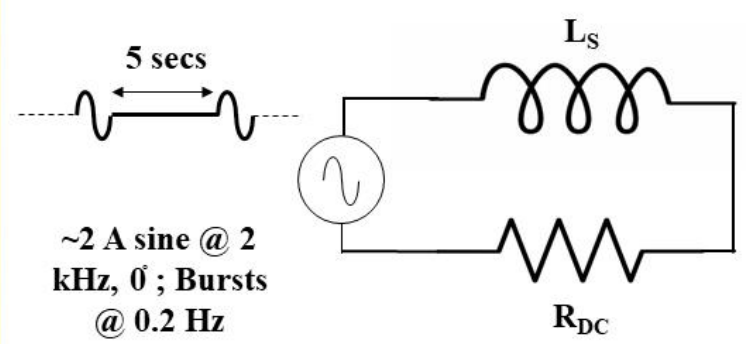

Figure 1. (a) MagPen prototyped in two different orientations of the $\mu$ coil: Type $\mathrm{H}(\mathrm{H}=$ horizontal) and Type V ( $\mathrm{V}=$ Vertical). The two insets show the zoomed in portion of the tips of MagPen for both Type $\mathrm{H}$ and Type $\mathrm{V}$ orientations. The $\mu$ coils are located the tip of the board and are coated with non-corrosive, biocompatible and insulation, Parylene-C. (b) The sub-mm $\mu$ coil compared to the size of a single rice grain. (c) Schematic of electrical equivalence of the $\mu$ coil as an RL circuit and diagram of the of the current waveform used drive the coil: 1-cycle of a sinusoid at $2 \mathrm{kHz}$ frequency, and $\sim 2 \mathrm{~A}$ in amplitude; each cycle of pulse is separated by 5 secs.

\subsection{Electromagnetic modeling}

Measuring the magnetic flux density and the induced electric field from these sub-mm sized $\mu$ coils is difficult experimentally difficult requiring custom-made miniature-sized pick-up coils [44] and close proximity of the $\mu$ coils. Reliable numerical modeling using finite element method (FEM)-based calculations to study induced currents in neural tissues have been previously reported to characterize both the electrical and magnetic fields [45-47]. Therefore, we conducted a FEM modeling study of the $\mu$ coil using ANSYS-Maxwell [48] eddy current solver (ANSYS, Canonsburg, PA, United States) which solves a modified version of the $\mathrm{T}-\Omega$ formulation of the Maxwell's equations [49]. The ceramic core $\mu$ coil dimensions, tissue slab parameters, boundary conditions and the high-resolution tetrahedral mesh size used are detailed in Table 1. Simulations were done using the Minnesota Supercomputing Institute (MSI) at the University of Minnesota ( 8 cores of Intel Haswell E5-2680v3 CPU, 64×8=512 GB RAM and 1 Nvidia Tesla K20 GPU). The induced electric field values were then exported to be analyzed using a customized code written in MATLAB (The Mathworks, Inc., Natick, MA, USA).

Table 1. Electromagnetic modeling parameters

\begin{tabular}{|c|c|}
\hline Parameter description & Value \\
\hline$\mu$ coil dimension $(\mathrm{L} \times \mathrm{W} \times \mathrm{H})$ & $1 \mathrm{~mm} \times 600 \mu \mathrm{m} \times 500 \mu \mathrm{m}$ \\
\hline no. of turns $(\mathrm{N})$ & 21 \\
\hline
\end{tabular}




\begin{tabular}{|c|c|}
\hline Wire diameter & $7 \mu \mathrm{m}$ \\
\hline Tissue dimension $(\mathrm{L} \times \mathrm{W} \times \mathrm{H})$ & $4 \mathrm{~mm} \times 4 \mathrm{~mm} \times 300 \mu \mathrm{m}$ \\
\hline Conductivity of tissue $(\sigma)$ & $0.13 \mathrm{~S} / \mathrm{m}$ \\
\hline Air dimension $(\mathrm{L} \times \mathrm{W} \times \mathrm{H})$ & $10 \mathrm{~mm} \times 10 \mathrm{~mm} \times 4 \mathrm{~mm}$ \\
\hline Energy error (user-specified) & $1 \%$ \\
\hline Final solution no. of mesh elements & 410,000 \\
\hline Adaptive passes (converged) & 6 \\
\hline
\end{tabular}

\subsection{Modeling using NEURON}

To simulate the effects of electric field induced by the magnetic field generated by the MagPen, we modified a model of a layer 5 (L5) pyramidal neuron developed by Pashut et al. [50]. In their model the spatial component of the induced electric filed generated by a transcranial magnetic stimulation (TMS) coil is projected into a $4 \mathrm{~mm} \times 4 \mathrm{~mm}$ array in MATLAB. Then simulations of the pyramidal neuron in the NEURON package [51,52] were performed at different positions of the neuron relative to the center of the coil (see section 3.2). We modified Pashut's NEURON [50] by replacing the array of the induced electric with ours generated from the FEM-model of the $\mu$ coils from ANSYS-Maxwell (see section 2.4). Simulations of the time varying waveform at $2 \mathrm{~A}$ amplitude sinusoidal current at $2 \mathrm{kHz}$ through the MagPen were carried out. The membrane potential at the soma was then measured and the volume of activation around the MagPen estimated.

\subsection{The hippocampal slice preparation and EPSP recording}

All brain slicing experiments (see Fig. 2(a)) were done in accordance with a protocol approved by the University of Minnesota Institutional Animal Care and Use Committee (IACUC). Brain slices were prepared at $300 \mu \mathrm{m}-400 \mu \mathrm{m}$ thickness (see Fig. 2(b) \& (c)) from 14- to 21-day-old Long-Evans (L/E) rats using a PELCO easiSlicerTM Vibratory Tissue Slicer (Ted Pella, Inc.). Slices were immediately incubated in standard aCSF with a composition (in $\mathrm{mM}$ ) of $124 \mathrm{NaCl}, 2 \mathrm{KCl}, 2 \mathrm{MgSO}_{4}, 1.25 \mathrm{NaH}_{2} \mathrm{PO}_{4}, 2 \mathrm{CaCl}_{2}, 26$ $\mathrm{NaHCO}_{3}$, and $10 \mathrm{D}$-glucose [53] at $33^{\circ} \mathrm{C}$ for at least 1 hour. The aCSF solution was oxygenated with a $95 \% \mathrm{O}_{2}$ and $5 \% \mathrm{CO}_{2}$ for the duration of the experiment. EPSPs were measured in the CA1 at $10 \mathrm{kHz}$ using a glass capillary microelectrode filled with aCSF (3-4 M 2 ) with a MultiClamp 700B Microelectrode Amplifier (Molecular Devices), Fig. 2(d). 


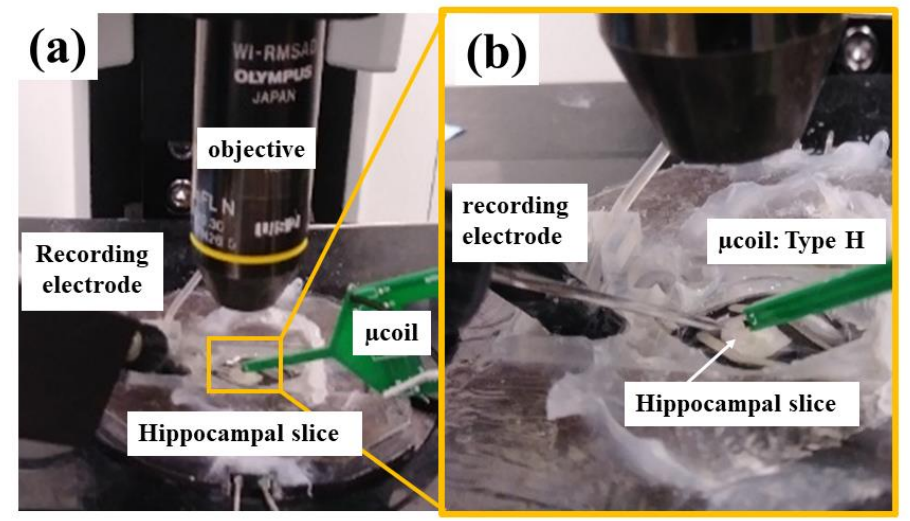

(d)
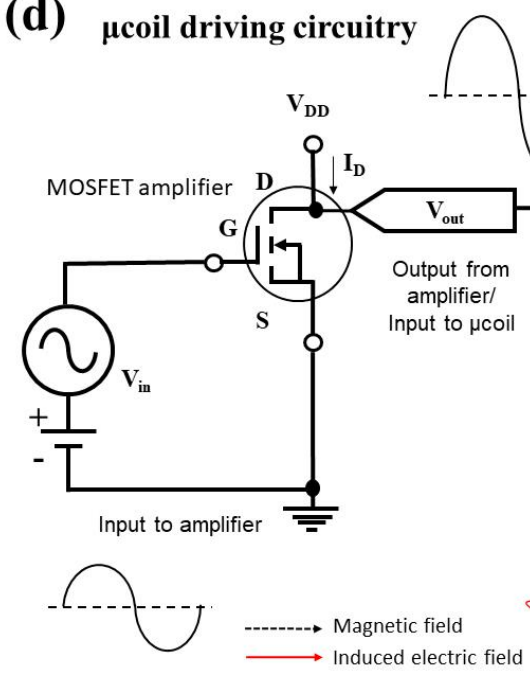

(c)

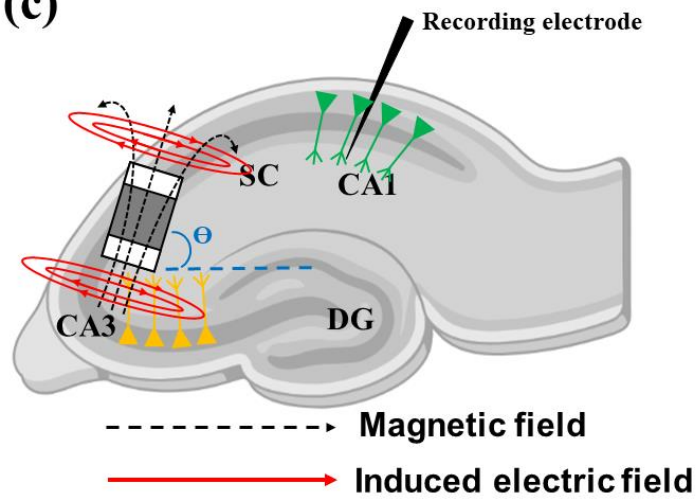

EPSP recording

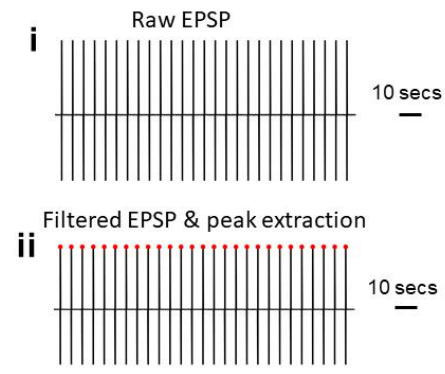

Averaged EPSP over each peak

iii

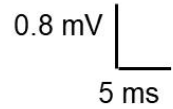

Figure 2. in vitro experimental set-up. (a) MagPen, Type-H orientated over the rat hippocampal slice for electrophysiological recordings. (b) Magnification of the chamber containing the hippocampal slice and $\mu$ coil in the preferred orientation, Type $\mathrm{H}$ for in vitro stimulation. (c) Orientation of the $\mu$ coil over Schaffer Collateral fibers which induces excitatory post synaptic potential (EPSP) from the CA1 neurons. (DC: Dentate Gyrus; CA3: Cornu Ammonis 3; SC: Schaffer Collateral; CA1: Cornu Ammonis 1). (d) $\mu$ coil driving circuitry (left): a function generator generating sinusoidal waveforms $\left(V_{\text {in }}\right)$ is amplified using a MOSFET amplifier with a user-controlled gain. The amplifier output $\left(\mathrm{V}_{\text {out }}\right)$ is used to drive the $\mu$ coil. EPSP recording (right): The raw EPSPs in (i) were recorded from the hippocampal slices. EPSPs were then analyzed through a customized algorithm MATLAB script to remove noise and estimate peak amplitude, an example of an EPSP is shown in (ii). Finally, EPSPs from approximately 20-25 trials were averaged to estimate the EPSP amplitude from CA1 neurons due to $\mu \mathrm{MS}$ over the SC fibers as in (iii).

\section{Results}

\subsection{Preferable $\mu$ coil orientation using modeling, position of neuron and validation using} experiments

As shown in Fig. 1(a), two prototypes for MagPen have been prepared in a horizontal (Type $\mathrm{H}$ ) and vertical (Type V) orientations. The ideal location and orientation of the coil activate the Schaffer Collateral fibers in the hippocampal slice (including the pyramidal cell layers) are shown in Fig. S2 (b) \& (c) in Supplementary Information S2. 
In Fig. 3(a) - i, ii \& iii, the spatial heat maps of the induced electric field from Type $\mathrm{H}$ orientation of the $\mu$ coil over a biological tissue at $300 \mu \mathrm{m}$ distance between tissue and the $\mu$ coil are shown. In Fig. 3(b) - i, ii \& iii, the spatial heat maps of the induced electric field from Type V orientation of the $\mu$ coil over a biological tissue at $300 \mu \mathrm{m}$ distance between tissue and the $\mu$ coil are shown. In both Fig. 3(a) \& (b), the $\mu$ coil is driven by a sinusoidal current of amplitude $2 \mathrm{~A}$ and frequency $2 \mathrm{kHz}$. The size of the neural tissue was $4 \mathrm{~mm} \times 4 \mathrm{~mm} \times 300 \mu \mathrm{m}$. The position of the modeled neuron is shown in Fig. 3(a) \& (b)- i, ii \& iii. To study the effects of micromagnetic neurostimulation, only the in-plane components, $\mathrm{E}_{\mathrm{x}}$ and $\mathrm{E}_{\mathrm{y}}$ of the induced electric field (E), were used in the model. Modeling the membrane potential from the neurons situated at a specified location (see Fig. 3(a) \& (b)) for both Type H and Type V oriented $\mu$ coils, action potentials were detected for the neuron being stimulated by Type $\mathrm{H}$ orientation only (see Fig. 3(a) - iv). For Type $\mathrm{V}$ orientation, the $\mathrm{E}_{\mathrm{x}}$-component of the induced electric field is negligible (see Fig. 3(b) - ii) failing to induce an action potential in the neuron (see Fig. 3(b) - iv).

In an ideal scenario, as demonstrated in Type $\mathrm{H}$ orientation in Fig. 3(a), the angle between the $\mu$ coil and the neural tissue were required to be $90^{\circ}$. However, in the in vitro experiments it was difficult to orient the Type $\mathrm{H}$ probe at an angle less than $90^{\circ}$. This was an unavoidable experimental challenge that created a difference between 'ideal' and 'real' orientation of Type H MagPen over the Schaffer collateral. In this in vitro experiment we recorded EPSPs from the CA1 region of rat hippocampal slices by stimulating the SC fibers using the MagPen. For the experimental set-up, Type H orientation (see Fig. 2(a) \& (b)) was found to elicit population EPSPs in neurons in the CA1.This observation was in corroboration with our FEMbased modeling studies done on ANSYS-Maxwell.

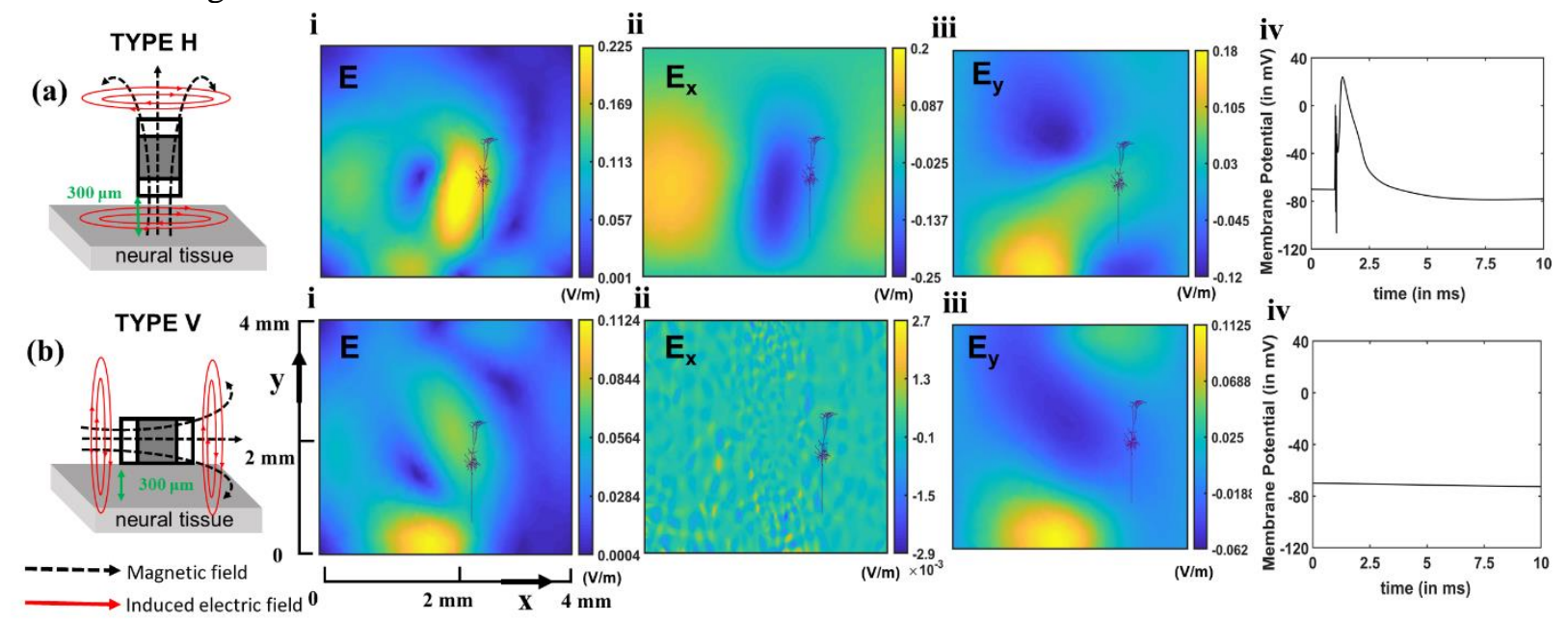

Figure 3. Modeling results corresponding to 'ideal' orientation of MagPen case for successful micromagnetic neurostimulation. The $\mu$ coil was driven by a sinusoidal current of amplitude $2 \mathrm{~A}$ at a frequency of $2 \mathrm{kHz}$. The approximate position of the modeled neuron with respect to the tissue has been shown. (a) MagPen, Type H: Spatial heat maps for the induced electric fields calculated for a tissue dimension of $4 \mathrm{~mm} \times 4 \mathrm{~mm} \times 300 \mu \mathrm{m}$, at $300 \mu \mathrm{m}$ from the $\mu$ coil surface for (i) $\mathrm{E}$ (in $\mathrm{V} / \mathrm{m}$ ) (ii) $\mathrm{E}_{\mathrm{x}}$ in $\mathrm{V} / \mathrm{m}$ (iii) $\mathrm{E}_{\mathrm{y}}$ in V/m (iv) membrane potential in the neuron; (b) MagPen, Type V: Spatial heat maps calculated for a tissue dimension of $4 \mathrm{~mm} \times 4 \mathrm{~mm} \times 300 \mu \mathrm{m}$ at $300 \mu \mathrm{m}$ from the $\mu$ coil surface for (i) $\mathrm{E}$ (in V/m) (ii) $E_{x}$ in $V / m$ (iii) $E_{y}$ in $V / m$ (iv) membrane potential in the neuron. Type $\mathrm{H}$ orientation in (a) elicited an action potential, but Type $\mathrm{V}$ orientation in (b) did not elicit an action potential. $\mathrm{x}$ and $\mathrm{y}$ in (b-i) denote the spatial axes coordinates in the neural tissue. It is same for all the spatial heatmaps.

\subsection{Spatial selectivity and distance dependence in micromagnetic neurostimulation}

For the Type $\mathrm{H}$ orientation of the MagPen, there were two unique features specific to micromagnetic neurostimulation observed. In Fig. 4(a)-i for the Type H orientation of the $\mu$ coil at a distance of $300 \mu \mathrm{m}$ from the neural tissue, activated approximately $2.672 \mathrm{~mm}^{2}$ of tissue. The most sensitive area of the tissue is marked by the ellipsoid in dotted black line where the $\mu$ coil position was at the center of the tissue marked 
by solid red line (see Fig. 4(a)-ii). On rotating the $\mu$ coil along any direction a completely new tissue area can be activated.

When the same Type H MagPen orientation was modeled, if the distance between the $\mu$ coil and neural tissue was greater than $300 \mu \mathrm{m}$, we observed a significant attenuation of the induced electric field (Fig. 4b). Validating the neurostimulation capability of this $\mu$ coil under this condition, the optimum distance for activation of a neural tissue was found to be $300 \mu \mathrm{m}$. Beyond this distance, no activation of the neuron was observed. Hence, in experiments on animal model, manipulating the correct distance between the $\mu$ coil and the neural tissue is extremely critical. The range of attenuation can be estimated from Fig. 4(b)-iii which is measured for distances between $\mu$ coil and tissue, $300 \mu \mathrm{m}, 500 \mu \mathrm{m}, 1000 \mu \mathrm{m}$, and $3000 \mu \mathrm{m}$ along the $\mathrm{x}$ axes of the neural tissue intersecting the y-axes at the center (see Fig. 4(b)-ii). A detailed demonstration of the attenuation of induced electric field (E) and its in-plane components $E_{x}$ and $E_{y}$ for distances between the tissue and $\mu$ coil above and below $300 \mu \mathrm{m}$ has been demonstrated in Supplementary Information S3.

In the simulation studies for both Fig. 4 (a) \& (b), the $\mu$ coil was powered by 2 A sinusoidal current in $2 \mathrm{kHz}$ frequency. The features of spatial selectivity and distance dependence of the $\mu$ coil are unique features for micromagnetic neurostimulation, which can potentially offer targeted, precise, highly focal, and spatially tunable neurostimulation capability.

(a)

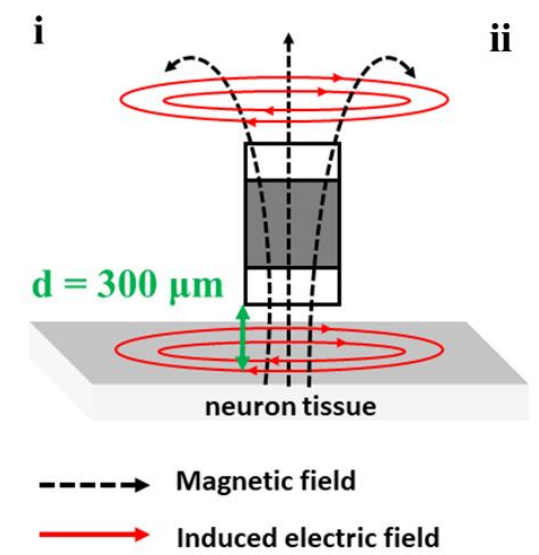

(b)

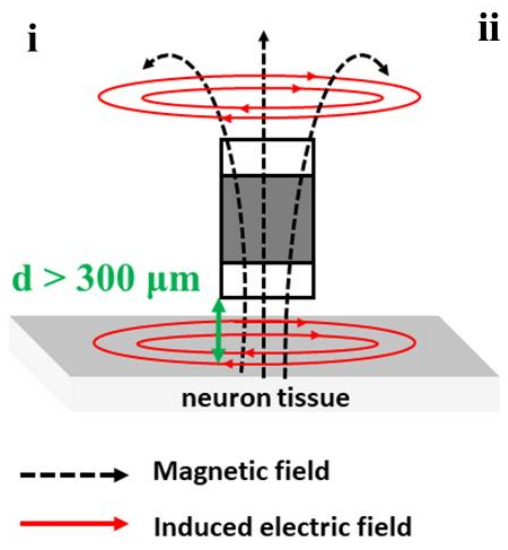

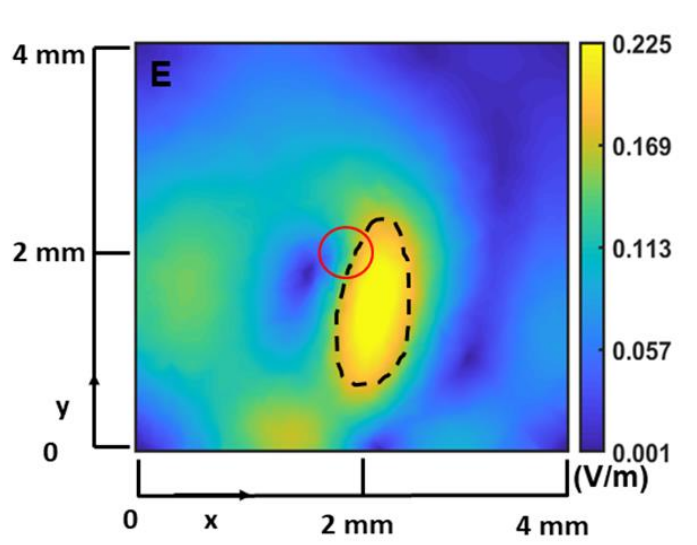

$4 \mathrm{~mm}$

Figure 4. Spatial selectivity and distance dependence of MagPen. (a) Spatial selectivity of MagPen, Type H. (i) For a distance, $d=300 \mu \mathrm{m}$ between the $\mu$ coil and the neural tissue, (ii) only $16.7 \%$ of the tissue area (denoted by the dotted black ellipsoid) has the maximum probability of getting stimulated. The location of the $\mu$ coil denoted by red solid circle. This implies that $\mu$ MS is spatially selective. (b) (i) For a distance, $d>$ $300 \mu \mathrm{m}$ between the Type $\mathrm{H}$ oriented $\mu$ coil and the neural tissue, (ii) the induced electric field value was 
measured along x-axes, along the dotted red line. (iii) As the distance between the $\mu$ coil and the neural tissue was increased, the induced electric field value decreased drastically. Our NEURON model showed that beyond $300 \mu \mathrm{m}$ distance between the $\mu$ coil and the neural tissue, no action potential was observed. The $\mu$ coil was driven by a $2 \mathrm{~A}$ sinusoidal current at $2 \mathrm{kHz}$ frequency. All measurements were made by varying the distance between the $\mu$ coil and the neural tissue of dimension $4 \mathrm{~mm} \times 4 \mathrm{~mm} \times 300 \mu \mathrm{m}$. $\mathrm{x}$ and $\mathrm{y}$ in (a-ii) $\&$ (b-ii) denote the spatial axes coordinates in the neural tissue. It is same for all the spatial heatmaps.

\subsection{Strength-frequency curve for micromagnetic neurostimulation}

We investigate the strength-frequency relationship which is the micromagnetic neurostimulation equivalent of the strength-duration relationship described for electrical stimulation. The induced electric field equation, which follows from Faraday's Law of electromagnetic induction, can be written as:

$$
\oint \boldsymbol{E}_{\text {ind }} \cdot d \boldsymbol{l}=-\iint \frac{\partial \boldsymbol{B}(\boldsymbol{t})}{\partial t} \cdot d \boldsymbol{S}
$$

where, $\boldsymbol{B}$ is the magnetic flux density generated from the $\mu$ coil, $\boldsymbol{E}_{\text {ind }}$ is the induced electric field that will stimulate the neurons, $\boldsymbol{l}$ and $\boldsymbol{S}$ are the contour and the surface area of the neural tissue.

In both our numerical calculations as well as in experiments we have used a sinusoidal current waveform to drive the $\mu$ coil. Therefore, the current waveform can be implemented as: $i(t)=I_{0} \sin (2 \pi f t)$, where, $I_{0}$ is the amplitude of the current, $f$ is the frequency of the current and $t$ is the time instant. The magnetic flux density is represented by: $B(t)=\frac{\mu_{r} \mu_{0} i(t) N}{L}$ where, $\mu_{r}$ is the relative permeability of the medium, $\mu_{0}$ is the vacuum permeability, $N$ is the number of turns of the $\mu$ coil and $L$ is the length of the $\mu$ coil. The spatial heatmap for the magnetic flux density $\left(B_{x, y, z}\right)$ is shown in Fig. S6 of Supplementary Information S6, whereas the temporal component of the magnetic flux density is equivalent to that of the current driving the $\mu$ coils.

On substituting the values for $i(t)$ and $B(t)$ in equation (1) followed by a simple differentiation, a modified version of induced electric field is obtained:

$$
\oint \boldsymbol{E}_{\text {ind }} \cdot d \boldsymbol{l}=-\frac{\mu_{r} \mu_{0} N}{L} \cdot I_{0} \cdot f \cdot 2 \pi \cdot \cos (2 \mu f t) \iint d \boldsymbol{S} \ldots(2) .
$$

Observing equation (2), we see that the parameters, $\mu_{r}, \mu_{0}, \boldsymbol{l}$ and $\boldsymbol{S}$ are all dependent on the neural tissue while, $N$ and $L$ are dependent on the $\mu$ coil. Both these groups of parameters cannot be altered and are specific to the neural tissue that we are trying to stimulate and the $\mu$ coil model that we are trying to use. There are only two parameters that we can alter, $I_{0}$ and $f$. $\boldsymbol{E}_{\text {ind }}$ is directly proportional to both $I_{0}$ and $f$. Equation (2) also demonstrates that the temporal component of the induced electric field will be a time derivative of the current driving the $\mu$ coils.

The working window obtained from numerical modeling on ANSYS-Maxwell in Fig. 4 shows that keeping the amplitude of the current constant at $2 \mathrm{~A}$, increasing the frequency of the current driving the $\mu$ coil from $500 \mathrm{~Hz}$ to $5 \mathrm{kHz}$, the induced electric field (E) amplitude also increases. This is evident from the spatial heat maps for $E, E_{x}$ and $E_{y}$ measured for a biological tissue of dimension $4 \mathrm{~mm} \times 4 \mathrm{~mm} \times 300$ $\mu \mathrm{m}$. The distance between the $\mu$ coil and the neural tissue has been $300 \mu \mathrm{m}$ in all cases. This working window in Fig. 5 implies that at higher frequency, the amplitude of the current required to drive the $\mu$ coils to trigger an action potential will be less. This means, at higher frequency, the $\mu$ coils will have low power consumption and this in terms of micromagnetic neurostimulation imply significant reduction of thermal effects on tissues.

Through subsequent numerical calculations on ANSYS-Maxwell [48] and NEURON [51] we plotted the 'strength-frequency' curve for micromagnetic neurostimulation specific for this $\mu$ coil model in Fig. 6. The heat maps at different sinusoidal current amplitudes and frequencies obtained from ANSYSMaxwell (eddy current solver), were input to the NEURON model. Then it was calculated at which current amplitude and frequency combination, the neuron elicits an action potential. Each of the points on the graph in Fig. 6 represent the current amplitude and frequency combination at which an action potential was observed. This implies that to elicit an action potential, at lower frequencies, we require a higher current amplitude and at higher frequency we require a lower current amplitude. However, in experiments (see 
section 3.4), the current amplitude required to stimulate a neuron at a certain frequency was found to be slightly greater because of some challenges discussed in section 4 in details.

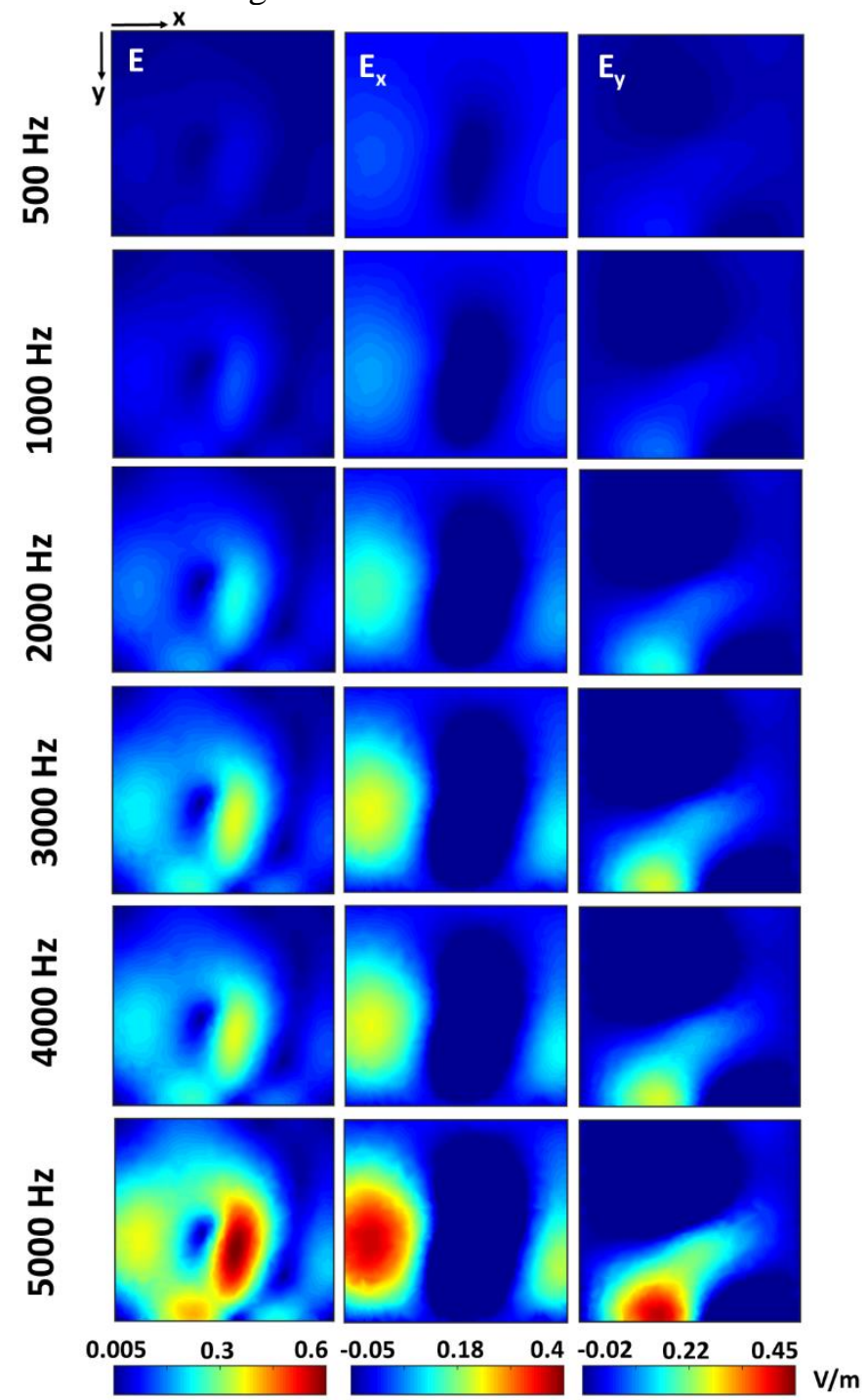

Figure 5. Effects of varying stimulation frequency on electric fields. Working window of the spatial heat maps of the $\mathrm{x}$-component $\left(\mathrm{E}_{\mathrm{x}}\right.$ in $\left.\mathrm{V} / \mathrm{m}\right)$ and the $\mathrm{y}$-component $\left(\mathrm{E}_{\mathrm{y}}\right.$ in $\left.\mathrm{V} / \mathrm{m}\right)$ of the induced electric $(\mathrm{E}$ in $\mathrm{V} / \mathrm{m})$ on neural tissue of dimension $4 \mathrm{~mm} \times 4 \mathrm{~mm} \times 300 \mu \mathrm{m}$ at different frequency of the $2 \mathrm{~A}$ of sinusoidal current driving the $\mu$ coil. With increasing frequency, the induced electric field value increased. From an experimental point-of-view, this suggests that at higher frequencies, the current amplitude required to stimulate a neuron should decrease. $\mathrm{x}$ and $\mathrm{y}$ denote the spatial co-ordinate axes directions of the tissue. 


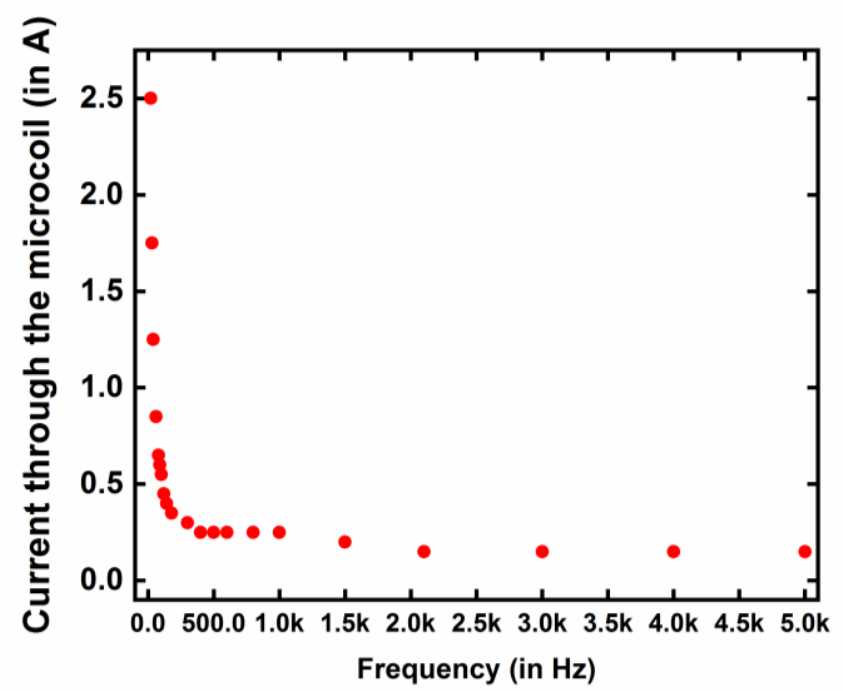

Figure 6. The strength-frequency curve for MagPen established through ANSYS-Maxwell and NEURON modeling.

\subsection{Validation of biological neuronal responses using TTX and TTX washout experiments}

In experiments, due to experimental limitations, it was not possible to place the $\mu$ coil in the 'ideal' orientation, as discussed later (see Fig. 2(c) \& Fig. S2(a), Supplementary Information S2). In this work, as shown in Fig. 2(d) in vitro EPSP recordings from CA1 region of the rat hippocampal slices were measured following stimulation of the Schaffer collaterals with the MagPen. The raw EPSPs were recorded, filtered, and averaged over at least 20-25 trials. Due to experimental challenges discussed in section 4 concerning spatial selectivity, distance dependence, and correct orientation of the MagPen prototype, and stimulus artifacts, EPSPs were validated by blocking the neural response using the voltage-gated Na channel blocker TTX [54]. The steps have been pictorially summarized in Supplementary Information S4. Because TTX blocks voltage gated $\mathrm{Na}$ channels, it blocks action potentials in Schaffer collaterals generated by the MagPen and the resulting EPSPs in CA1 are eliminated. After washing out the TTX we would expect a complete, or at least a partial, recovery of the EPSP in response to magnetic stimulation. Fig. 7(a)-i shows the EPSP recorded from CA1by MagPen Type H stimulation on the Schaffer collaterals. Without altering the position of the MagPen and the EPSP recording electrode, the slice was superfused with artificial CSF containing $1 \mu \mathrm{M}$ of TTX for $35-40$ mins. At this time, the magnetic stimulation was applied, and the recording is shown in Fig. 7(a)-ii. Finally, response after 10 minute TTX washout is shown in Fig. 7(a)-iii.

The superposition of the EPSPs measured before, during TTX and after washout are shown in Fig 7(b). It can be seen that the EPSP (blue trace, from Fig. 7(a)-i) disappeared after application of TTX (orange trace, from Fig 7(a)-ii). This suggests that the signal induced by magnetic was dependent on neuronal action potentials. After washout of TTX the trough observed before TTX was almost completely recovered (yellow trace, from Fig. 7(a)-iii). These results support the conclusion that the signal observed is an EPSP and not an electronic artifact. 
(a)
(i)

stim

(ii)

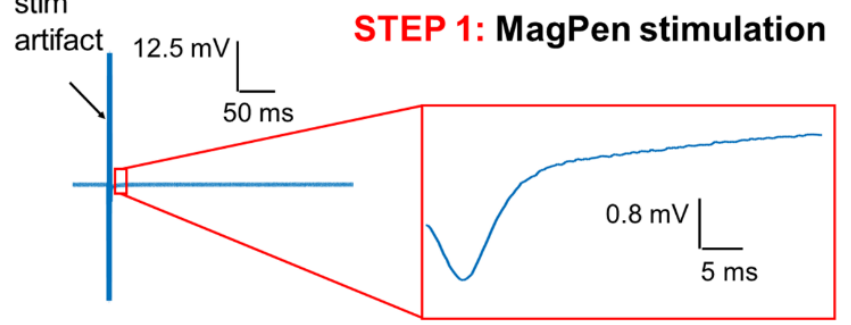

(iii)

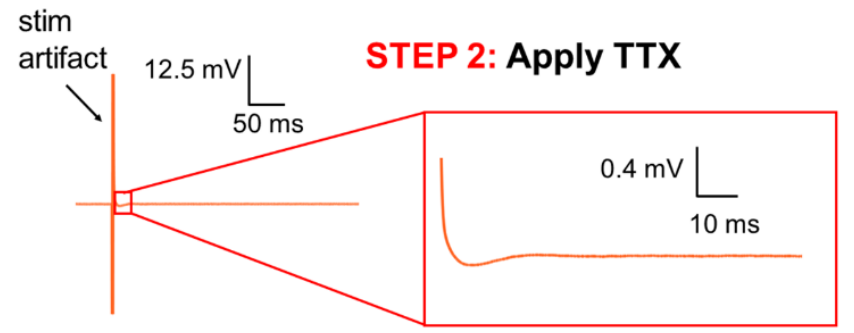

\section{stim}
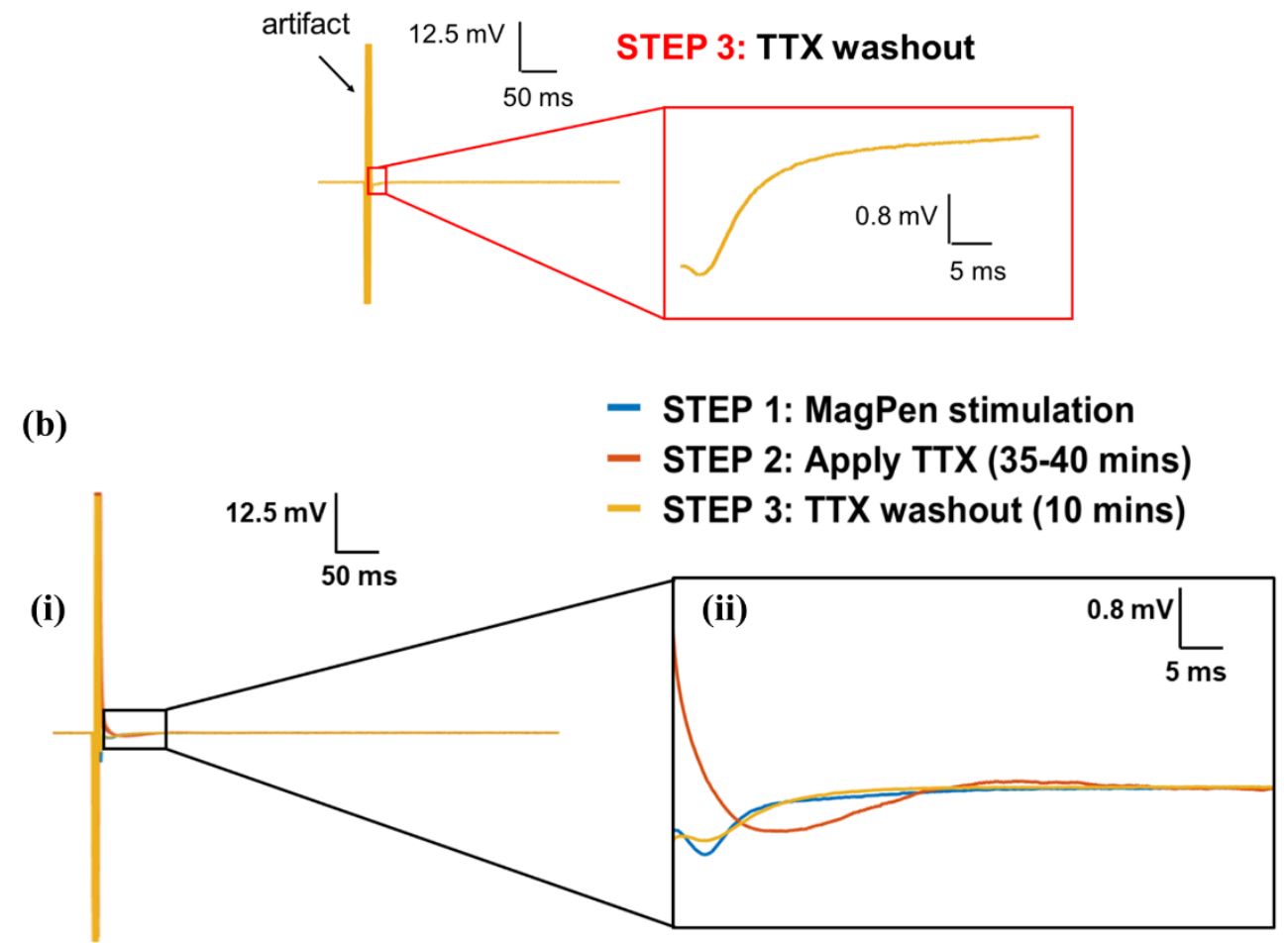

Figure 7. Validating whether the EPSP recording from MagPen stimulation is a biological response. In all cases, MagPen powered by 2 A @ 2 kHz sinusoidal pulse @ 5 secs. The MagPen stimulation was applied at the CA3 region and the EPSP was recorded at the CA1 region. The EPSP recorded from: (a)-(i) STEP 1 - MagPen stimulation only: Main EPSP of the averaged signal is zoomed out by omitting the stimulation artifact. (ii) STEP 2 - Apply TTX (35 - 40 mins): To the same hippocampal slice, $1 \mu \mathrm{M}$ of TTX was perfused at a rate of $0.8-1 \mathrm{ml} / \mathrm{min}$ for $35-40 \mathrm{mins}$. Main EPSP of the averaged signal is zoomed out by omitting the stimulation artifact. (iii) STEP 3 - TTX washout (10 mins): To the same hippocampal slice, TTX was washed out by perfusing aCSF solution at a rate of $0.8-1 \mathrm{ml} / \mathrm{min}$ for $10 \mathrm{mins}$. Main EPSP of the averaged signal is zoomed out by omitting the stimulation artifact. (b) Overlapped EPSPs obtained from the 3 steps in (a). (b)-(i) Averaged EPSPs overlapped in STEP 1, STEP 2 and STEP 3. (ii) The average signals zoomed in, obtained by removal of the stimulation artifact. The removal of the trough from STEP 1 (trace in blue) in STEP 2 (trace in orange) implies that TTX successfully blocked the neuron response in 
STEP 1. In addition, the signal observed in STEP 1 is indeed biological. Successful TTX washout in STEP 3 can be concluded from the partial reappearance of the trough in STEP 3 (trace in yellow). This further strengthens the fact that the trace in blue obtained from magnetic stimulation only is indeed biological. This was observed in 4 out of 6 trials of this 3 -step experiment.

\section{Discussion}

Micromagnetic neurostimulation is still in its infancy with limited biologically meaningful results. However, the involvement of several research groups worldwide in this line of research has made it increasingly popular. Three unique features of this technology that had been previously identified, have been quantified in this work: (1) orientation dependence, (2) spatial selectivity and, (3) depth dependence.

First, with respect to orientation dependence, there has been enough controversy in the literature regarding what orientation is the most suitable to observe elicitation of a neuron response, in both in vitro and in vivo settings. Bonmassar et al. [29], Lee et al. [55] and Osanai et al. [56] have performed independent calculations and experiments to arrive at a necessary conclusion. While Bonmassar et al. [29] in their very first works mentions how both orientations can elicit a neuron response that can be recorded using patch clamp set-up, Lee et al. [55] mentions that Type $\mathrm{H}$ orientation over the pyramidal neurons is second most preferable option to observe a neuron response with Type $\mathrm{V}$ orientation being the most preferable. On the contrary, Osanai et al. [56] in their in vivo study on cortical neurons mentions how they observed local field potentials (LFP) using Type H orientation only on the cortex up to a significant depth of $950 \mu \mathrm{m}$. In this work, we corroborate with Osanai et al.'s [56] work to conclude that Type H orientation is the preferable option to elicit a neuron response (see section $3.1 \& 3.4$ ). However, further research studies are encouraged to establish this point because the performance of micromagnetic neurostimulation can vary between different population of neurons in both in vitro and in vivo studies. It might also be highly dependent on the orientation of the neurons in the experimental study settings. Hence, preliminary studies involving this technology should consider a well investigated neuron model in experiments (with known electric field threshold and frequency) as in this work we used the well oriented Schaffer Collateral fibers of the hippocampal slice as the experimental model.

Second, the quantified spatial selectivity of these $\mu$ coils demonstrated in Fig. 4(a) for this $\mu$ coil model is reported for the first time in the literature of micromagnetic neurostimulation. We demonstrated that a particular position of this $\mu$ coil model driven by a 2 A sinusoidal current waveform at $2 \mathrm{kHz}$ frequency activates $2.672 \mathrm{~mm}^{2}$ of the tissue area can be stimulated at a time. Rotating the $\mu$ coil along any direction clockwise or anticlockwise - will activate a completely different $2.672 \mathrm{sq} \mathrm{mm}$ of the tissue area. Hence, during the in vitro experiments to record EPSPs from hippocampal slices, care had to be taken that at a time one does not accidentally rotate the MagPen prototype in the micromanipulator. This fact also justifies why we could not validate the EPSPs from 2 trials out of 6 trials in the 3-step TTX-TTX washout experiments demonstrated in Section 3.4 and Supplementary Information S5. The amount of tissue area that is expected to be activated will vary with the dimension of the $\mu$ coil and the distance between the $\mu$ coil and the tissue.

Third, this work numerically established the distance dependence of micromagnetic stimulation and the threshold distance for this $\mu$ coil model. The numerical simulations suggested that it was essential to maintain the distance between the $\mu$ coil and the tissue well below $300 \mu \mathrm{m}$. Beyond $300 \mu \mathrm{m}$ our modeling results from the NEURON model showed no neurostimulation effect (see Supplementary Information S3). Therefore, this distance dependent feature for micromagnetic neurostimulation promises targeted and local activation of neurons. As much as this feature is the ardent need for next generation neuromodulation devices, this also makes EPSP recordings from micromagnetic neurostimulation extremely challenging. During our in vitro experiments, it was essential to keep the distance between $\mu$ coil and the neural tissue well below $300 \mu \mathrm{m}$. This also means we must control the thickness of the Parylene-C coating on the $\mu$ coil. While using the micromanipulator to adjust the distance between the $\mu$ coil and the hippocampal tissue, it is critical to adjust the distance between the $\mu$ coil and the tissue below $300 \mu \mathrm{m}$.

In Section 3.3, the strength-frequency curve for this $\mu$ coil model has been established using ANSYS-Maxwell and NEURON modeling. In experiments (discussed in section 3.4) the threshold current required to drive the $\mu$ coil such that we can stimulate the CA3 neurons (marked in yellow in Fig. 2(c)) at 2 
$\mathrm{kHz}$ frequency is $\sim 2 \mathrm{~A}$. However, the strength-frequency curve in Fig. 6 reads $\sim 0.25 \mathrm{~A}$. The reason behind the higher threshold in experiments can be justified by the challenges faced during the in vitro experimental set-up in Fig. 2(a) related to orientation dependence, spatial selectivity and distance sensitivity. If the $\mu$ coil model is changed, the trend might still be the same, but the numerical values will vary.

We chose hippocampal slices because the well aligned axons from CA3 projecting to the CA1 region through the Schaffer collateral (see Fig. 2(c)) make this a common preparation for electrophysiological experiments. Furthermore, seizures have been induced in hippocampus in that past, making it a suitable subject for studying epilepsy [57]. Although this technology supports spatially selective neurostimulation, we did not face difficulty in deciding which set of neurons to record from. As we chose to study the CA3-CA1 hippocampal synaptic pathway, we kept the recording electrode fixed in the CA1 region. On stimulating the CA3 region using MagPen prototype, one could record EPSPs from the synaptically activated CA1 neurons (marked in green in Fig. 2(c)) if the Type H prototype was oriented at a distance below $300 \mu \mathrm{m}$ between the $\mu$ coil and the tissue.

Reproducibility for in vitro experiments related to micromagnetic neurostimulation is a pressing issue due to several challenges related to electromagnetic interference (EMI), spatial selectivity and distance dependence. Nevertheless, with tedious grounding efforts and the correct adjustment of MagPen prototype orientation over the hippocampal tissue we have been able to obtain biological EPSPs from micromagnetic stimulation. The EPSPs could be suppressed by application of TTX in 4 out of 6 trials with an average of $20 \%$ drop in the EPSP peak (see Supplementary Information S5). We also observed partial return of EPSPs on TTX washout with a success rate in 2 out of 3 trials of successful suppression on TTX application (see Supplementary Information S5).

There is ample research scope in this interesting yet challenging field of research. One can envision development of a portable, robust, and user-friendly waveform generator system [58] specific to drive these $\mu$ coils such that micromagnetic neurostimulation can be translated to be studied in a clinical setting. Besides, the development of custom-fabricated $\mu$ coils [35] in order to lower the power of operation and make the induced electric field even more spatially focused will be an interesting way to proceed with development in this field. In addition, it would be interesting to know how arrays of $\mu$ coils [34] can spatially activate different populations of neurons. The high distance sensitivity with which these magnetic $\mu$ coils can stimulate the neurons has been studied. At the same time, it has been predicted how miniscule these $\mu$ coils can be fabricated to facilitate cellular level neurostimulation [34,35]. Hence, for this technology of micromagnetic neurostimulation to reach clinical settings, it requires advancement in neurosurgical techniques too. Finally, since these $\mu$ coils use magnetic field to stimulate the neurons, there is ample scope of using a wide variety of waveforms to stimulate the neurons unlike electrical electrodes where due to safety limitations, one cannot use all types of waveforms for neurostimulation. Advancement in $\mu$ coil designs promises implantable neuromodulation that may complement or be an alternative to electrical stimulation.

\section{Conclusion}

This work tests a novel micromagnetic coil for neuromodulation in a hippocampal slice. Only one orientation of the $\mu$ coil, Type H-MagPen prototype, could successfully activate neurons; hence they demonstrate orientation-specific activation. For the first time we have quantified that a tissue area of 2.672 $\mathrm{mm}^{2}$ can be activated at a time for a particular location of this $\mu$ coil model. This contributes to spatially selective stimulation capability of the $\mu$ coils. We investigated this using numerical simulations and corroborated through in vitro EPSP recording experiments that this $\mu$ coil at 2 A sinusoidal current of frequency $2 \mathrm{kHz}$ cannot activate neurons beyond a $\mu$ coil-tissue distance of $300 \mu \mathrm{m}$. The EPSPs averaged over 20-25 trials from the micromagnetic stimulation were validated to be successful biological responses using a previously reported TTX. On application of TTX, the EPSPs dropped on an average of 20\% (calculated over 4 successful trials out of 6). On TTX washout, partial return of the EPSPs was observed; the difference between original EPSP peak and the peak after TTX washout is $0.1 \%$ on an average. Finally, we demonstrated the strength-frequency curve specific for MagPen which showed that at higher frequency of the driving current, lower current amplitudes are required to activate the neurons. 


\section{ORCID ID}

Renata Saha: 0000-0002-0389-0083

Sadegh Faramarzi: 0000-0002-9739-9286

Robert P. Bloom: 0000-0002-7781-5270

Kai Wu: 0000-0002-9444-6112

Denis Tonini: 0000-0001-5121-5544

Susan A. Keirstead: 0000-0002-7610-678X

Theoden I. Netoff: 0000-0002-0115-1930

Jian-Ping Wang: 0000-0003-2815-6624

\section{Notes}

The authors declare no conflict of interest.

\section{Acknowledgements}

This study was financially supported by the Minnesota Partnership for Biotechnology and Medical Genomics under award number ML2020. Chap 64. Art I, Sec11on 4. R.S. acknowledge the 3-year College of Science and Engineering (CSE) Fellowship awarded by University of Minnesota, Twin Cities. The authors would also like to thank useful discussions Dr. Winfried A. Raabe, M.D. from the Department of Neurosurgery; Kendall H. Lee, M.D., PhD, Charles D. Blaha, PhD and Yoonbae Oh, PhD from Mayo Clinic, Rochester, MN. Portions of this work were conducted in the Minnesota Nano Center (MNC), which is supported by the National Science Foundation through the National Nano Coordinated Infrastructure Network (NNCI) under Award Number ECCS-2025124. J.P.W also thanks the Robert Hartmann Endowed chair support.

\section{References}

[1] Bourbeau D, Creasey G, French J, Grill W M, Howley S, Krassioukov A, Moritz C, Santacruz B, Thor $\mathrm{K}$ and Wheeler $\mathrm{T} 2020$ A roadmap for advancing neurostimulation approaches for bladder and bowel function after spinal cord injury Spinal Cord $\mathbf{5 8}$ 1227-32

[2] Marjenin T, Scott P, Bajaj A, Bansal T, Berne B, Bowsher K, Costello A, Doucet J, Franca E and Ghosh C 2020 FDA perspectives on the regulation of neuromodulation devices Neuromodulation: Technology at the Neural Interface 23 3-9

[3] Gurgu E, Gurgu I-A and Tonis R B M 2020 Neuromarketing for a better understanding of consumer needs and emotions Independent Journal of Management \& Production 11 208-35

[4] Dones I and Levi V 2018 Spinal cord stimulation for neuropathic pain: current trends and future applications Brain Sciences $\mathbf{8} 138$

[5] Kapural L, Gupta M, Paicius R, Strodtbeck W, Vorenkamp K E, Gilmore C, Gliner B, Rotte A, Subbaroyan J and Province-Azalde R 2020 Treatment of chronic abdominal pain with 10-kHz spinal cord stimulation: safety and efficacy results from a 12-month prospective, multicenter, feasibility study Clinical and translational gastroenterology $\mathbf{1 1}$

[6] Formento E, Minassian K, Wagner F, Mignardot J B, Le Goff-Mignardot C G, Rowald A, Bloch J, Micera S, Capogrosso M and Courtine G 2018 Electrical spinal cord stimulation must preserve proprioception to enable locomotion in humans with spinal cord injury Nature neuroscience 21 1728-41

[7] Xu W, Zhang C, Deeb W, Patel B, Wu Y, Voon V, Okun M S and Sun B 2020 Deep brain stimulation for Tourette's syndrome Translational neurodegeneration 9 1-19

[8] Kaminska M, Perides S, Lumsden D E, Nakou V, Selway R, Ashkan K and Lin J-P 2017 Complications of deep brain stimulation (DBS) for dystonia in children-the challenges and 10 year experience in a large paediatric cohort European Journal of Paediatric Neurology 21 168-75

[9] Amon A and Alesch F 2017 Systems for deep brain stimulation: review of technical features Journal of Neural Transmission 124 1083-91 
[10] Moisset X, Lanteri-Minet M and Fontaine D 2019 Neurostimulation methods in the treatment of chronic pain Journal of Neural Transmission 1-14

[11] Galhardoni R, Correia G S, Araujo H, Yeng L T, Fernandes D T, Kaziyama H H, Marcolin M A, Bouhassira D, Teixeira M J and de Andrade D C 2015 Repetitive transcranial magnetic stimulation in chronic pain: a review of the literature Archives of physical medicine and rehabilitation 96 S156-72

[12] Dahlhamer J, Lucas J, Zelaya C, Nahin R, Mackey S, DeBar L, Kerns R, Von Korff M, Porter L and Helmick C 2018 Prevalence of chronic pain and high-impact chronic pain among adults-United States, 2016 Morbidity and Mortality Weekly Report 671001

[13] Nguyen S, Cloutier F, Philippon D, Côté M, Bussieres R and Backous D D 2016 Outcomes review of modern hearing preservation technique in cochlear implant Auris Nasus Larynx 43 485-8

[14] Lupo J E, Biever A and Kelsall D C 2020 Comprehensive hearing aid assessment in adults with bilateral severe-profound sensorineural hearing loss who present for Cochlear implant evaluation American journal of otolaryngology 41102300

[15] Wong C L, Ching T Y, Cupples L, Button L, Leigh G, Marnane V, Whitfield J, Gunnourie M and Martin L 2017 Psychosocial development in 5-year-old children with hearing loss using hearing aids or cochlear implants Trends in Hearing 212331216517710373

[16] Perez-Malagon C D and Lopez-Gonzalez M A 2021 Epilepsy and Deep Brain Stimulation of Anterior Thalamic Nucleus Cureus $\mathbf{1 3}$

[17] Vingerhoets F J, Villemure J-G, Temperli P, Pollo C, Pralong E and Ghika J 2002 Subthalamic DBS replaces levodopa in Parkinson's disease: two-year follow-up Neurology 58 396-401

[18] Seaton B T, Hill D F, Cowen S L and Heien M L 2020 Mitigating the Effects of Electrode Biofouling-Induced Impedance for Improved Long-Term Electrochemical Measurements In Vivo Analytical chemistry 92 6334-40

[19] Shenai M B, Falconer R and Rogers S 2019 A Cupriavidus Pauculus Infection in a Patient with a Deep Brain Stimulation Implant Cureus 11

[20] Xu J and Lee H 2020 Anti-biofouling strategies for long-term continuous use of implantable biosensors Chemosensors $\mathbf{8} 66$

[21] Rolston J D, Englot D J, Starr P A and Larson P S 2016 An unexpectedly high rate of revisions and removals in deep brain stimulation surgery: analysis of multiple databases Parkinsonism \& related disorders 33 72-7

[22] Falowski S M and Bakay R A 2016 Revision surgery of deep brain stimulation leads Neuromodulation: Technology at the Neural Interface $19443-50$

[23] Mohsin S A, Sheikh N M and Saeed U 2008 MRI induced heating of deep brain stimulation leads: Effect of the air-tissue interface Progress In Electromagnetics Research 83 81-91

[24] Sonmez A I, Camsari D D, Nandakumar A L, Voort J L V, Kung S, Lewis C P and Croarkin P E 2019 Accelerated TMS for depression: a systematic review and meta-analysis Psychiatry research 273 $770-81$

[25] Roth Y, Tendler A, Arikan M K, Vidrine R, Kent D, Muir O, MacMillan C, Casuto L, Grammer G and Sauve W 2021 Real-world efficacy of deep TMS for obsessive-compulsive disorder: post-marketing data collected from twenty-two clinical sites Journal of Psychiatric Research 137 667-72

[26] Short G, Rosen L, Liu J, Hegarty B, Szegda J, Westphal C, Cermak J and Wessel T 20171164 THE USE OF CHEMICAL NEURO STIMULATION TO MINIMIZE SLEEP DISTURBANCE ASSOCIATED WITH MUSCLE CRAMPING AND SPASTICITY Journal of Sleep and Sleep Disorders Research $40 \mathrm{~A} 434-5$

[27] Yoo S, Lee H, Jun S B, Kim Y-K and Ji C-H 2018 Disposable MEMS optrode array integrated with single LED for neurostimulation Sensors and Actuators A: Physical 273 276-84

[28] Menz M D, Ye P, Firouzi K, Nikoozadeh A, Pauly K B, Khuri-Yakub P and Baccus S A 2019 Radiation force as a physical mechanism for ultrasonic neurostimulation of the ex vivo retina Journal of Neuroscience 39 6251-64

[29] Bonmassar G, Lee S W, Freeman D K, Polasek M, Fried S I and Gale J T 2012 Microscopic magnetic stimulation of neural tissue Nature communications $\mathbf{3} 921$ 
[30] Gimsa J, Habel B, Schreiber U, van Rienen U, Strauss U and Gimsa U 2005 Choosing electrodes for deep brain stimulation experiments-electrochemical considerations Journal of neuroscience methods 142 251-65

[31] Campbell A and Wu C 2018 Chronically implanted intracranial electrodes: tissue reaction and electrical changes Micromachines 9430

[32] Lee S W, Fallegger F, Casse B D and Fried S I 2016 Implantable microcoils for intracortical magnetic stimulation Science advances 2 e1600889

[33] Lee S W, Thyagarajan K and Fried S I 2018 Micro-Coil Design Influences the Spatial Extent of Responses to Intracortical Magnetic Stimulation IEEE Transactions on Biomedical Engineering 66 168094

[34] Rizou M-E and Prodromakis T 2018 Magnetic stimulation in the microscale: The development of a $6 \times 6$ array of micro-coils for stimulation of excitable cells in vitro Biomedical Physics \& Engineering Express 4025016

[35] Jeong H, Deng J and Bonmassar G 2021 Planar figure-8 coils for ultra-focal and directional micromagnetic brain stimulation Journal of Vacuum Science \& Technology B, Nanotechnology and Microelectronics: Materials, Processing, Measurement, and Phenomena 39063202

[36] Park H-J, Bonmassar G, Kaltenbach J A, Machado A G, Manzoor N F and Gale J T 2013 Activation of the central nervous system induced by micro-magnetic stimulation Nature communications 4 2463

[37] Lee S W and Fried S I 2014 The response of L5 pyramidal neurons of the PFC to magnetic stimulation from a micro-coil 2014 36th Annual International Conference of the IEEE Engineering in Medicine and Biology Society (IEEE) pp 6125-8

[38] Bonmassar G and Serano P 2020 MRI-Induced Heating of Coils for Microscopic Magnetic Stimulation at 1.5 Tesla: An Initial Study Frontiers in human neuroscience 1453

[39] Saha R, Wu K, Su D and Wang J-P 2019 Tunable magnetic skyrmions in spintronic nanostructures for cellular-level magnetic neurostimulation Journal of Physics D: Applied Physics

[40] Su D, Wu K, Saha R and Wang J-P 2019 Tunable magnetic domain walls for therapeutic neuromodulation at cellular level: Stimulating neurons through magnetic domain walls Journal of Applied Physics 126183902

[41] Wu K, Su D, Saha R and Wang J-P 2019 Spin-Orbit Torque and Spin Hall Effect-Based Cellular Level Therapeutic Spintronic Neuromodulator: A Simulation Study The Journal of Physical Chemistry C $12324963-72$

[42] Bi G and Poo M 2001 Synaptic modification by correlated activity: Hebb's postulate revisited Annual review of neuroscience 24 139-66

[43] Bi G and Poo M 1998 Synaptic modifications in cultured hippocampal neurons: dependence on spike timing, synaptic strength, and postsynaptic cell type Journal of neuroscience 18 10464-72

[44] Minusa S, Osanai H and Tateno T 2017 Micromagnetic stimulation of the mouse auditory cortex in vivo using an implantable solenoid system IEEE Transactions on Biomedical Engineering 65 1301-10

[45] Wagner T, Gangitano M, Romero R, Théoret H, Kobayashi M, Anschel D, Ives J, Cuffin N, Schomer D and Pascual-Leone A 2004 Intracranial measurement of current densities induced by transcranial magnetic stimulation in the human brain Neuroscience letters 354 91-4

[46] Wagner T A, Zahn M, Grodzinsky A J and Pascual-Leone A 2004 Three-dimensional head model simulation of transcranial magnetic stimulation IEEE Transactions on Biomedical Engineering 51 158698

[47] Golestanirad L, Mattes M, Mosig J R and Pollo C 2010 Effect of model accuracy on the result of computed current densities in the simulation of transcranial magnetic stimulation IEEE Transactions on Magnetics 46 4046-51

[48] Martyanov A S and Neustroyev N I 2014 ANSYS Maxwell Software for electromagnetic field calculations Eastern European Scientific Journal

[49] Ren Z $2002 \mathrm{~T}$-/spl omega/formulation for eddy-current problems in multiply connected regions IEEE Transactions on Magnetics 38 557-60 
[50] Pashut T, Wolfus S, Friedman A, Lavidor M, Bar-Gad I, Yeshurun Y and Korngreen A 2011 Mechanisms of magnetic stimulation of central nervous system neurons PLoS Comput Biol 7 e1002022

[51] Hines M L and Carnevale N T 1997 The NEURON simulation environment Neural computation 9 1179-209

[52] Carnevale N T and Hines M L 2006 The NEURON book (Cambridge University Press)

[53] Moyer Jr J R and Brown T H 1998 Methods for whole-cell recording from visually preselected neurons of perirhinal cortex in brain slices from young and aging rats Journal of neuroscience methods $\mathbf{8 6}$ $35-54$

[54] Lipowsky R, Gillessen T and Alzheimer C 1996 Dendritic Na+ channels amplify EPSPs in hippocampal CA1 pyramidal cells Journal of neurophysiology 76 2181-91

[55] Lee S W and Fried S I 2016 Enhanced control of cortical pyramidal neurons with micromagnetic stimulation IEEE Transactions on Neural Systems and Rehabilitation Engineering 25 1375-86

[56] Osanai H, Minusa S and Tateno T 2018 Micro-coil-induced Inhomogeneous Electric Field Produces sound-driven-like Neural Responses in Microcircuits of the Mouse Auditory Cortex In Vivo Neuroscience $371346-70$

[57] Netoff T I and Schiff S J 2002 Decreased neuronal synchronization during experimental seizures Journal of Neuroscience 22 7297-307

[58] Dong L, Li G, Tian C, Lin L, Gao Y and Zheng Y 2021 Design of Submillimeter Magnetic Stimulation Instrumentation and Its Targeted Inhibitory Effect on Rat Model of Epilepsy IEEE Transactions on Instrumentation and Measurement 70 1-8 
${ }^{y}$

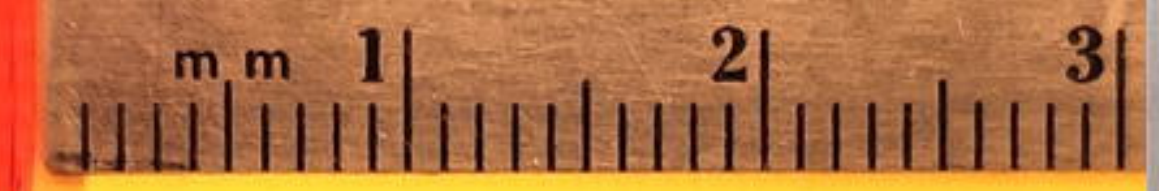

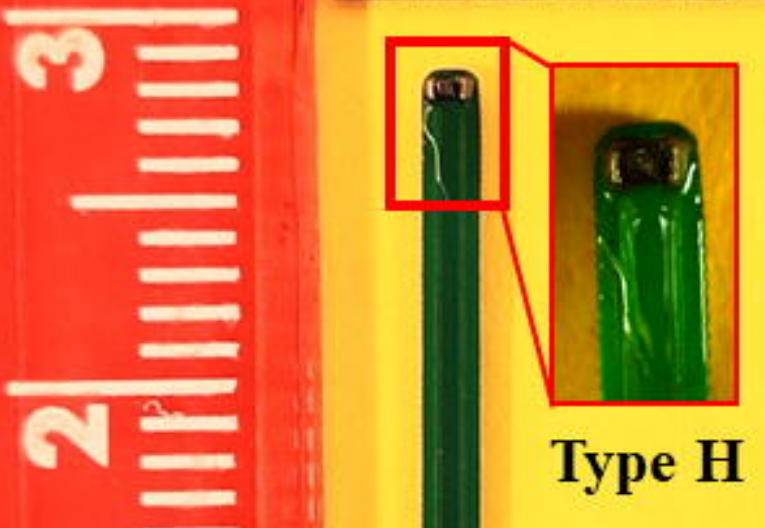

mones

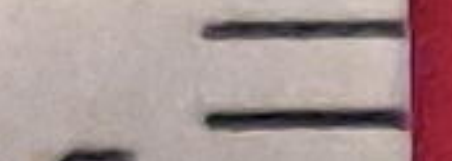

E

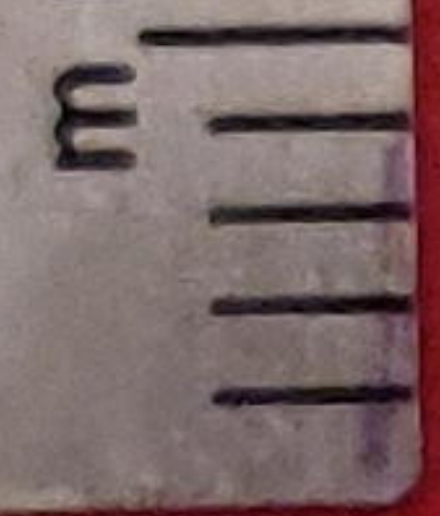

Type V

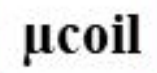

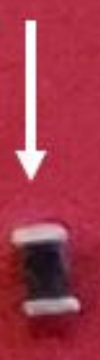

$1 \mathrm{~mm}$

(c)

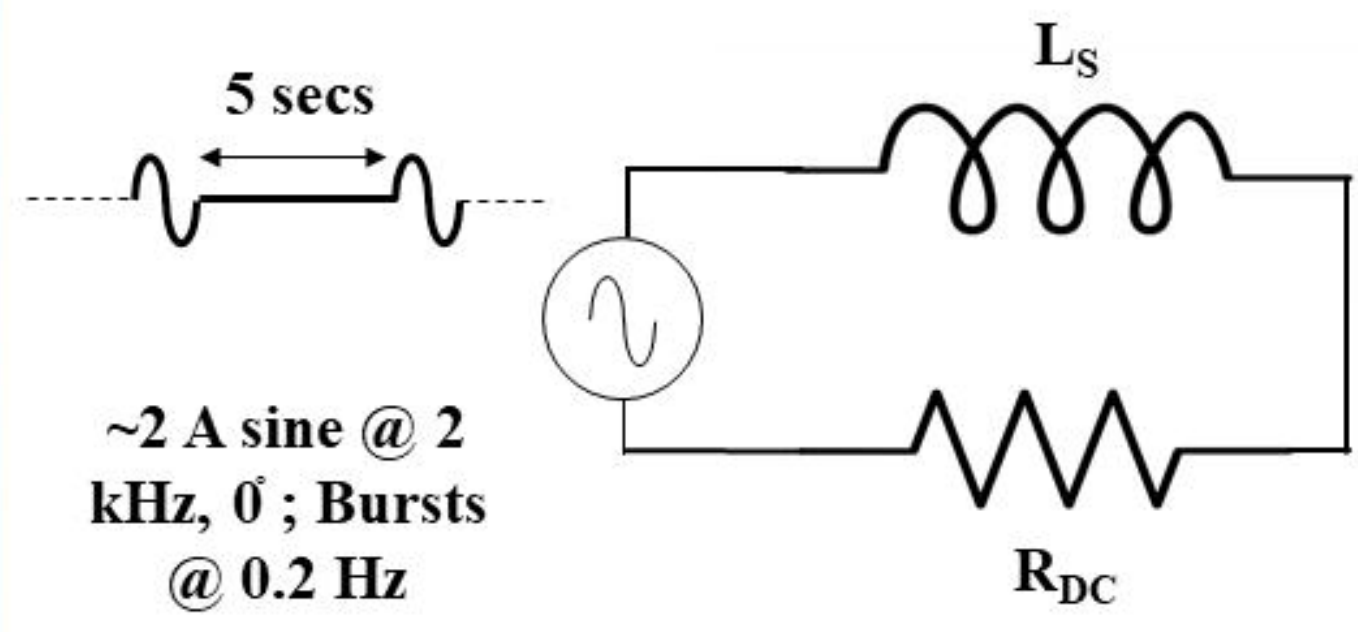




\section{TYPE H}

(a)

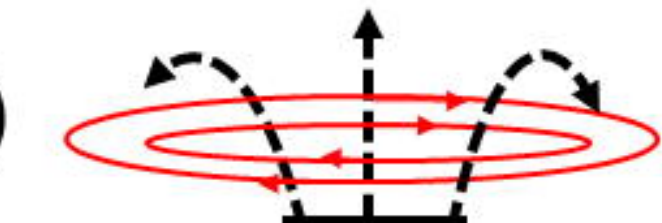

neural tissue

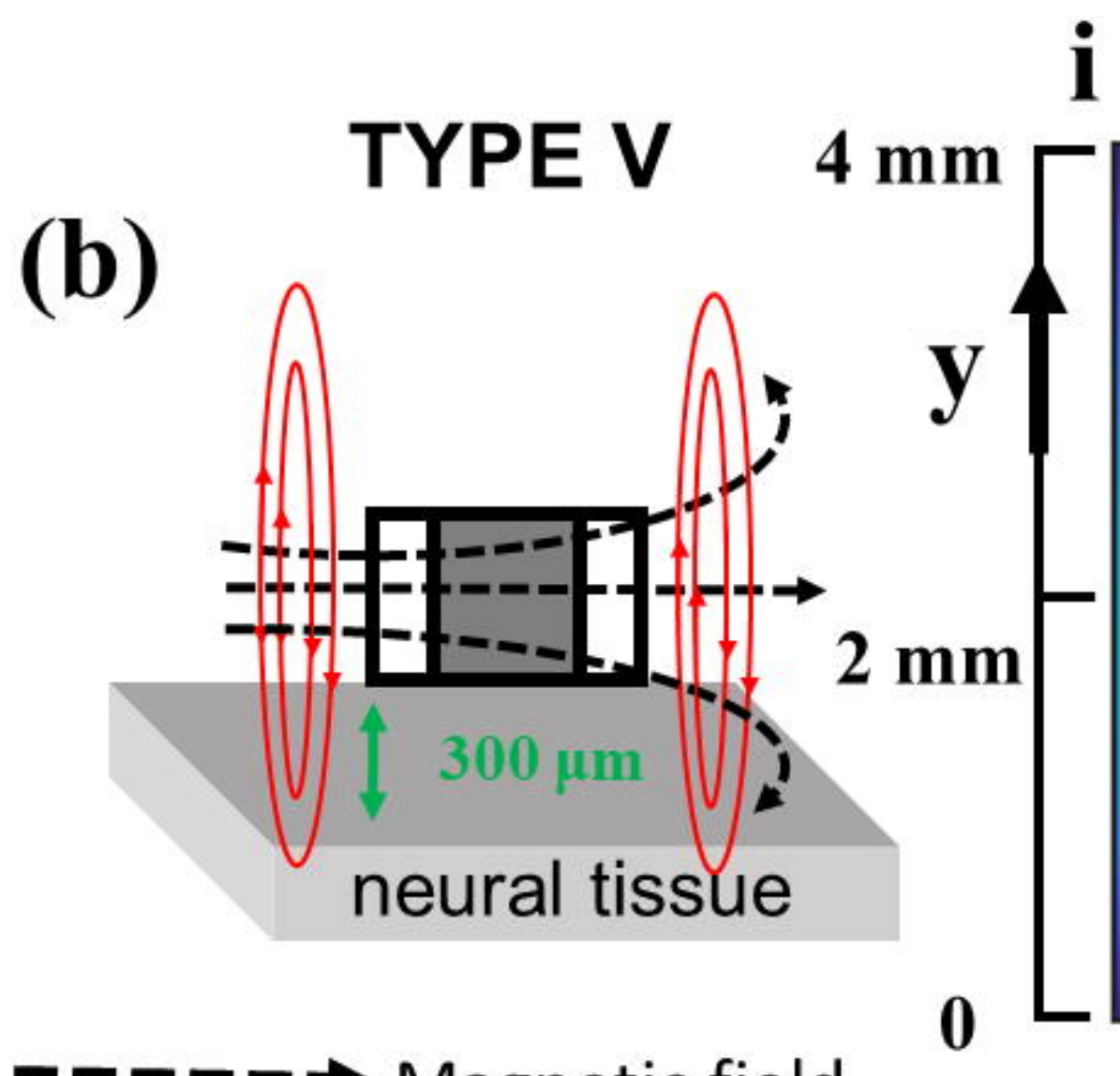

Magnetic field Induced electric field ${ }^{0}$ i $\quad$ ii

iii

iv

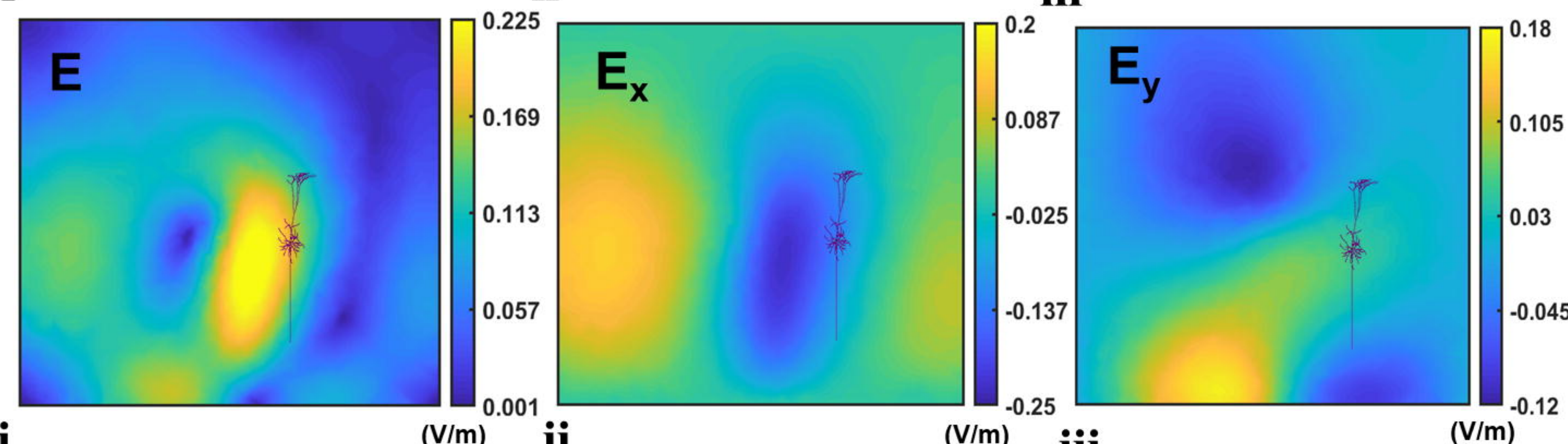

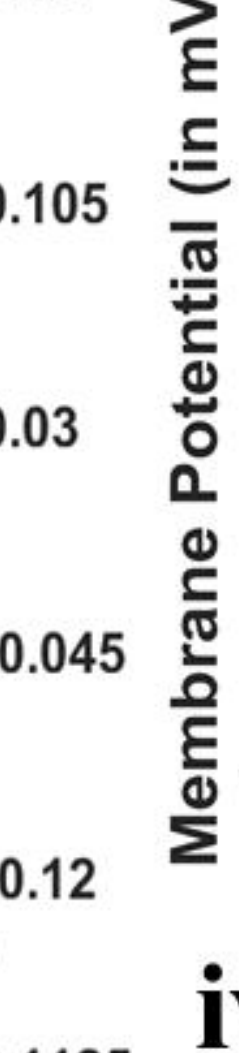

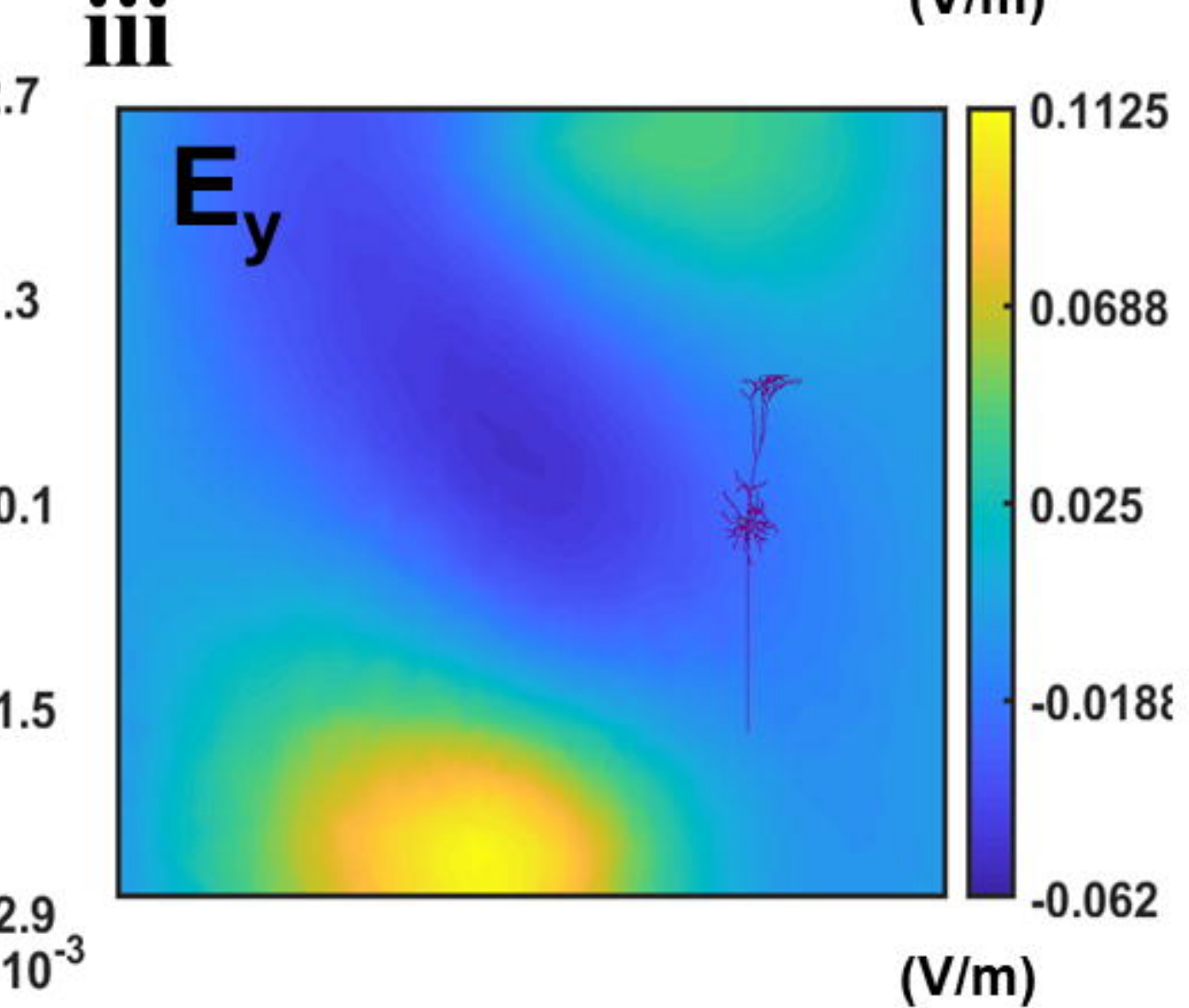

iv

time (in ms)

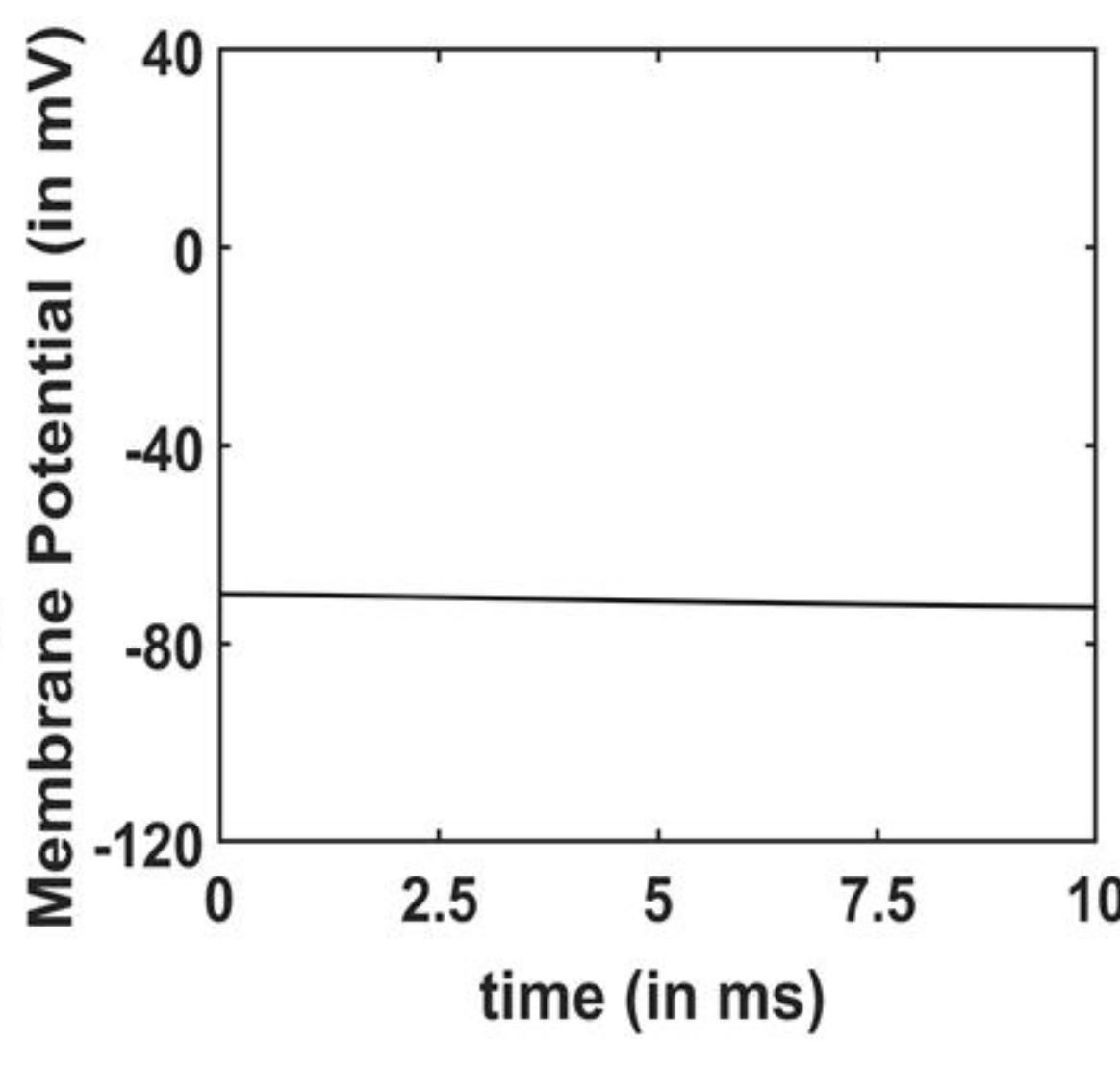




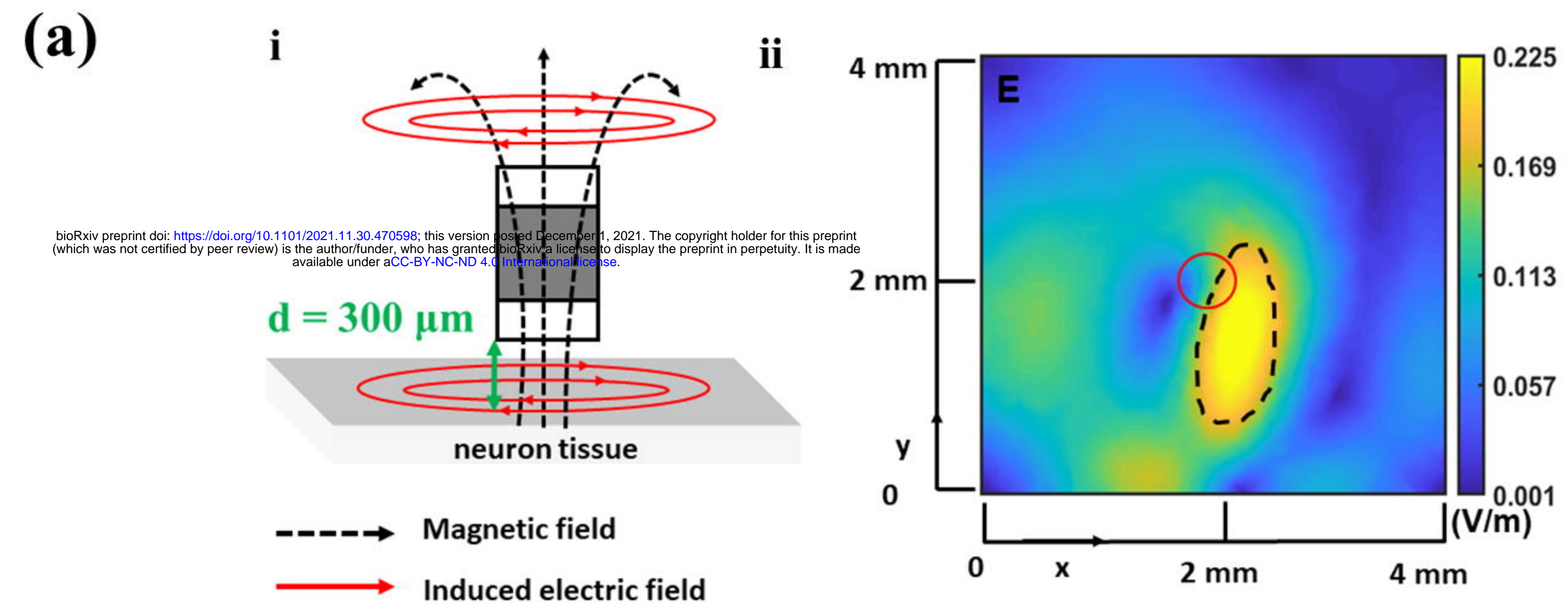

(b)

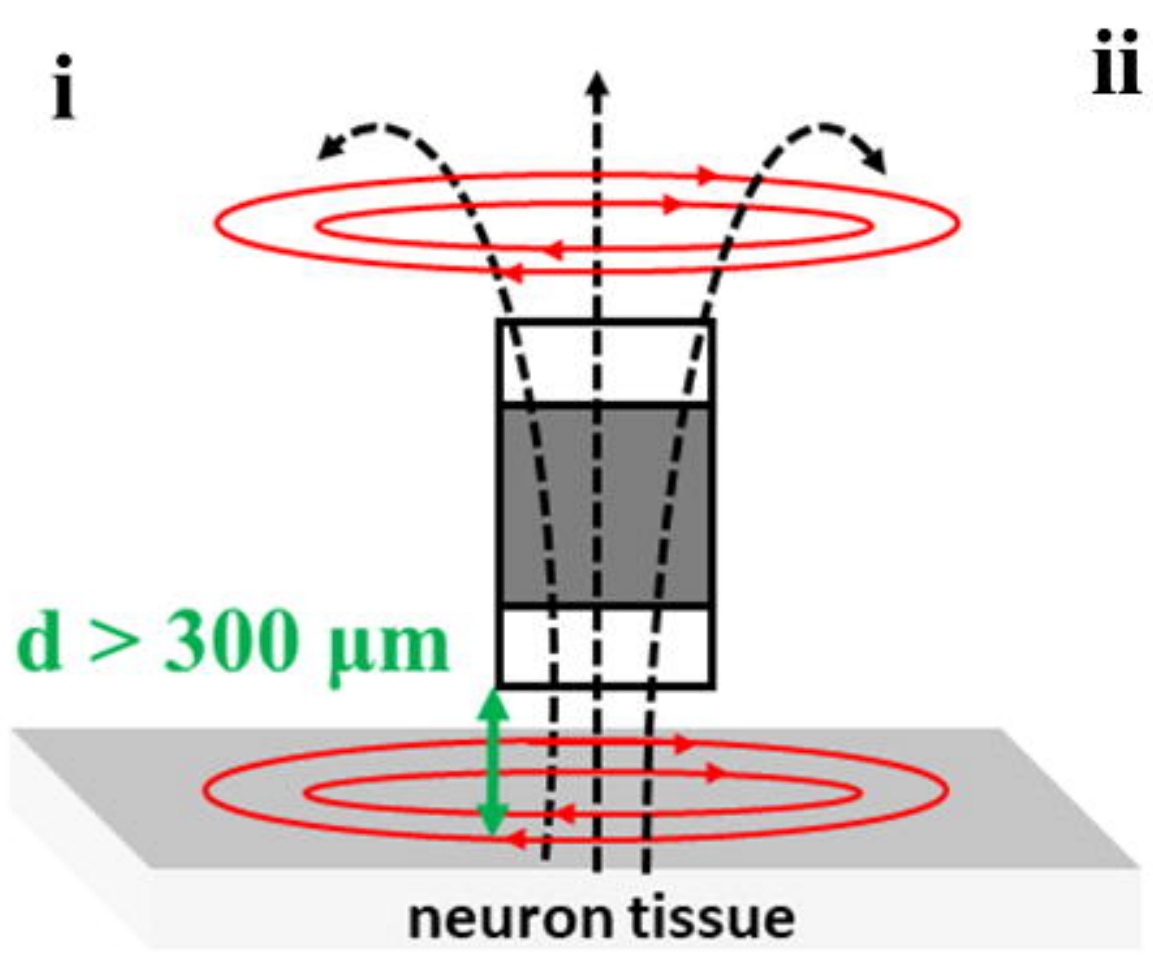

Magnetic field

Induced electric field

ii

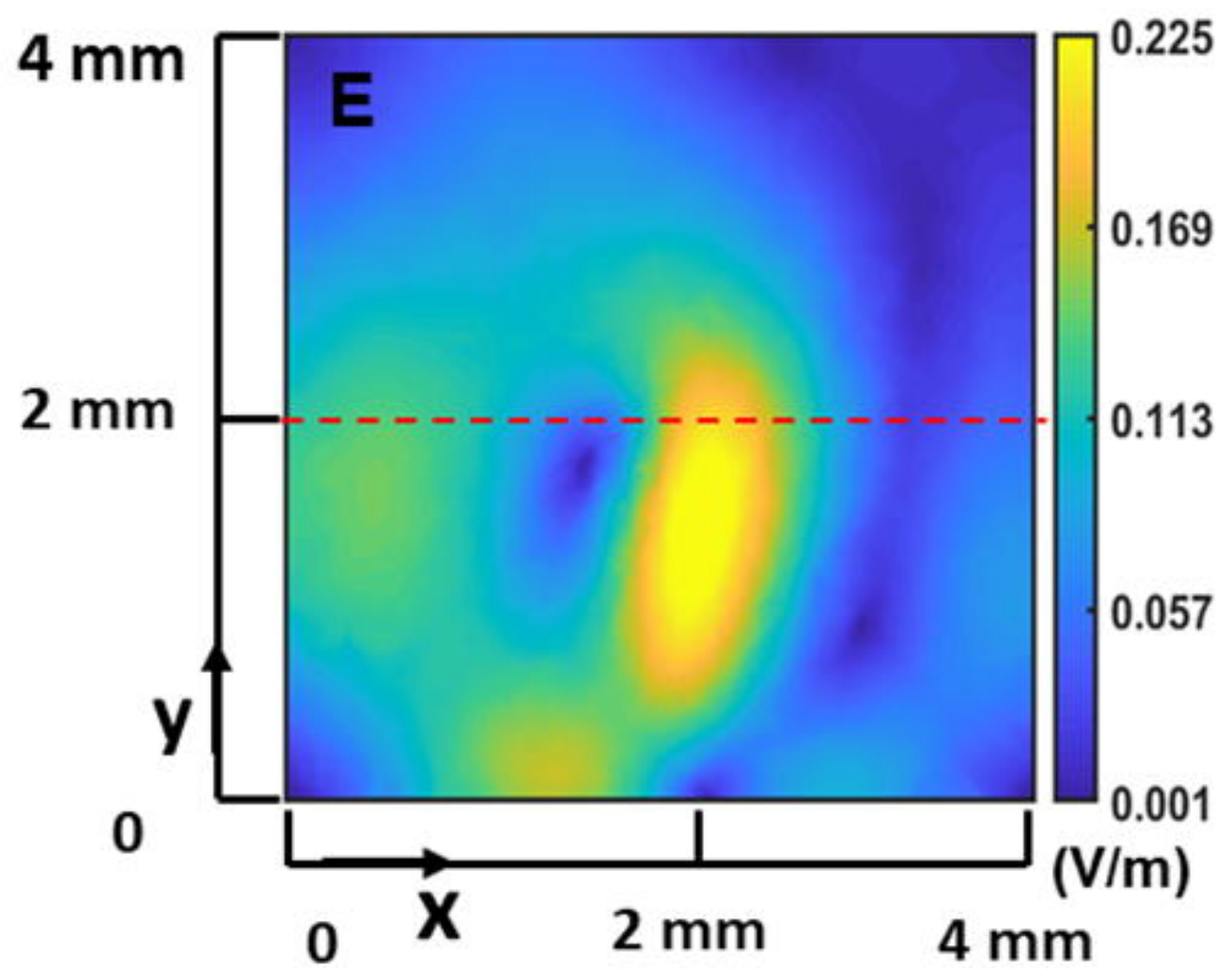

iii

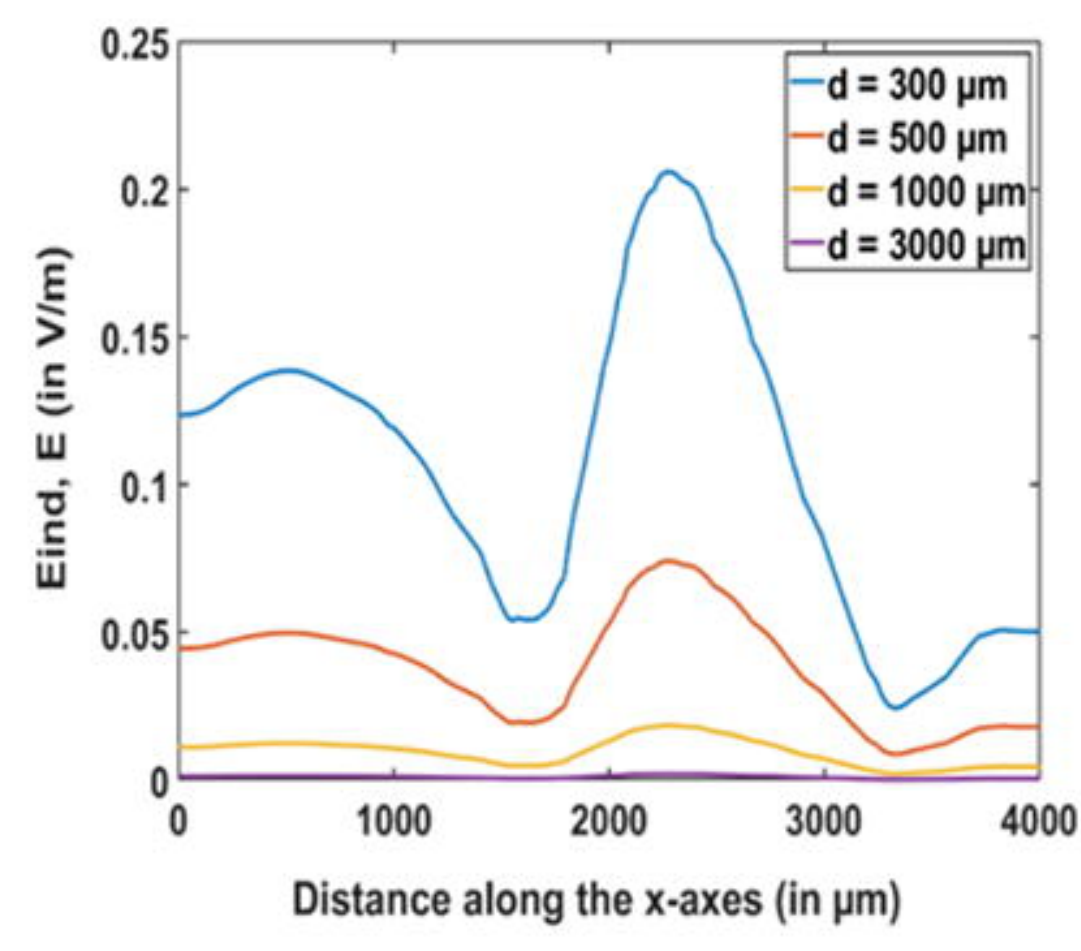




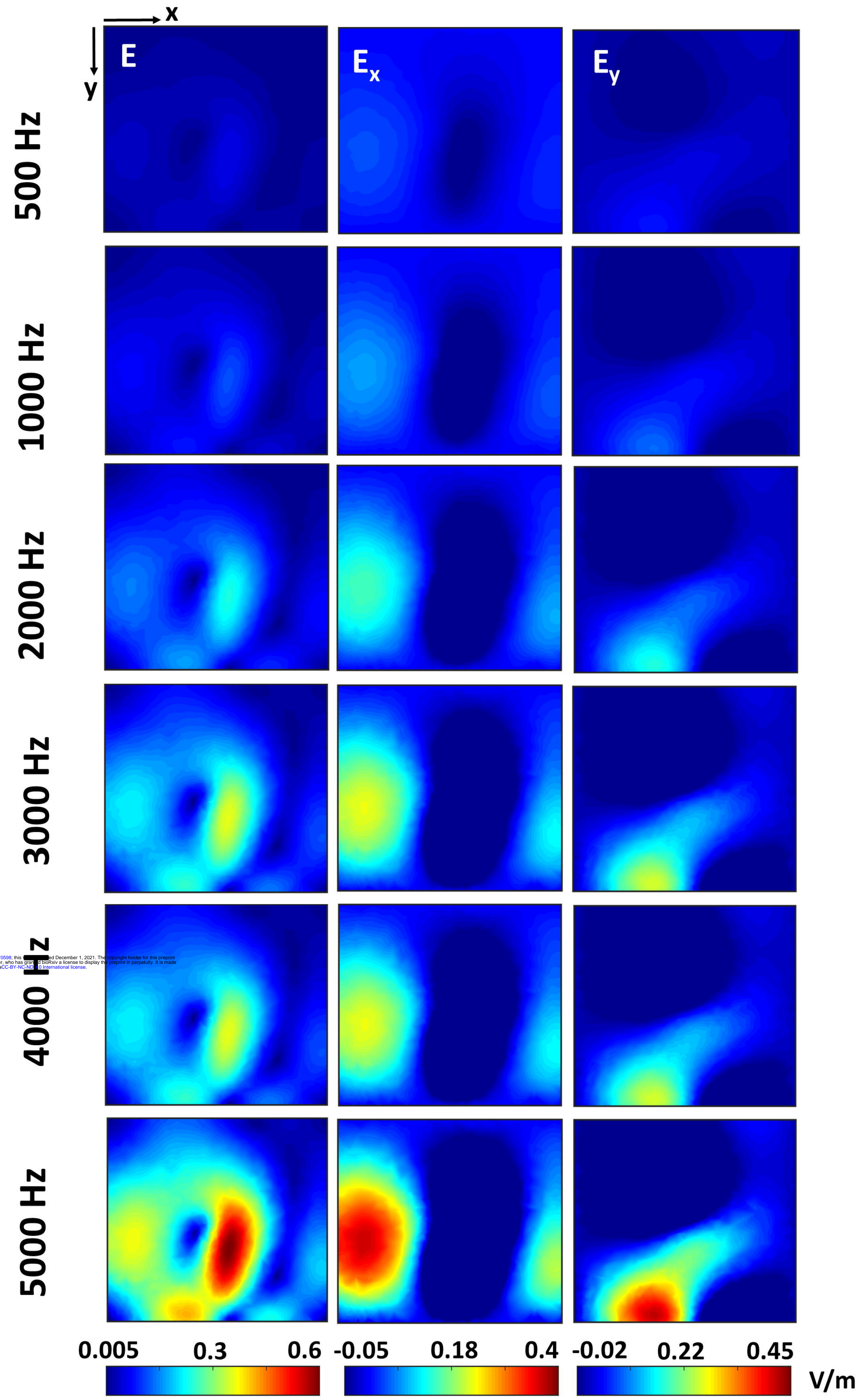




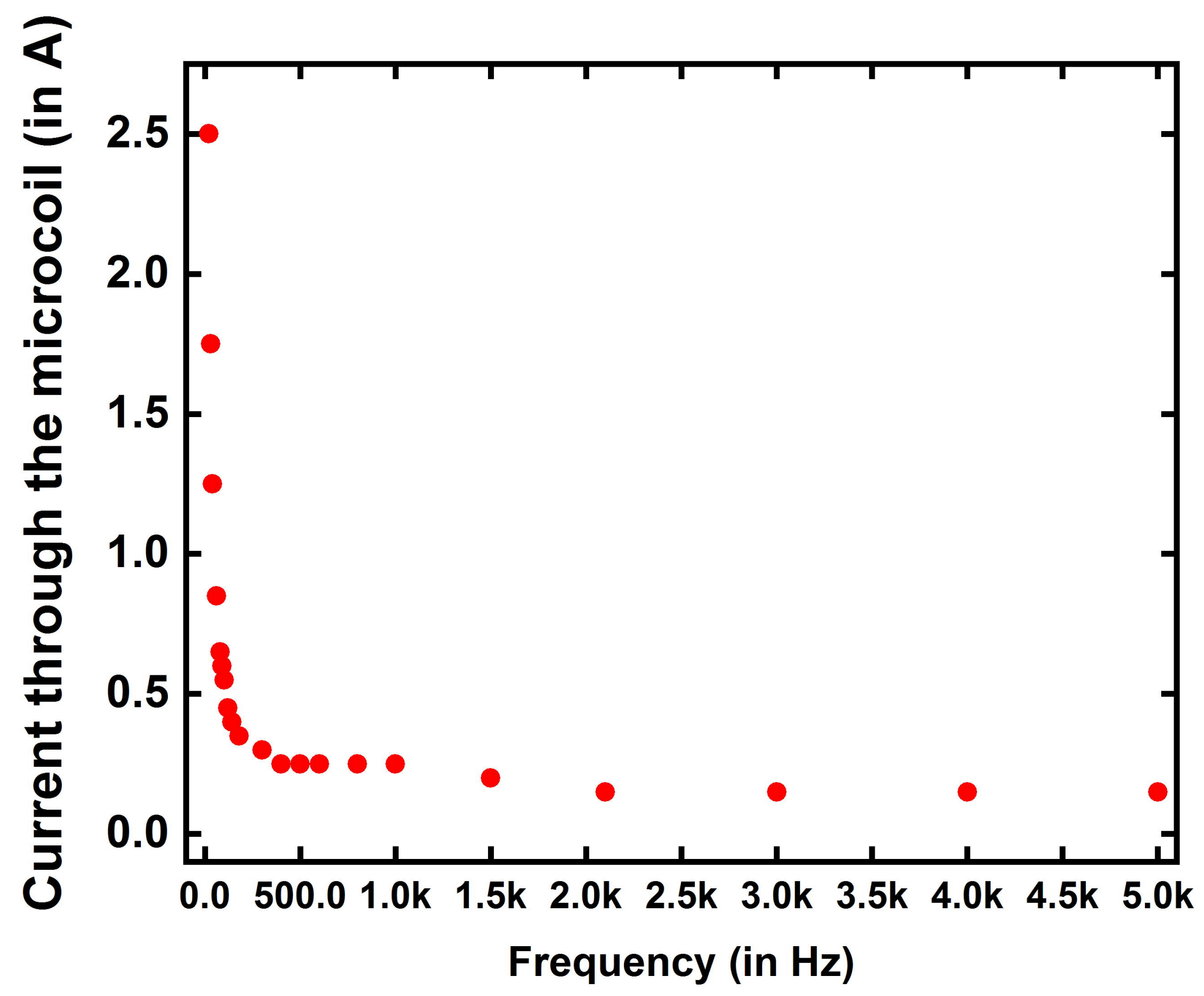


(a)

stim

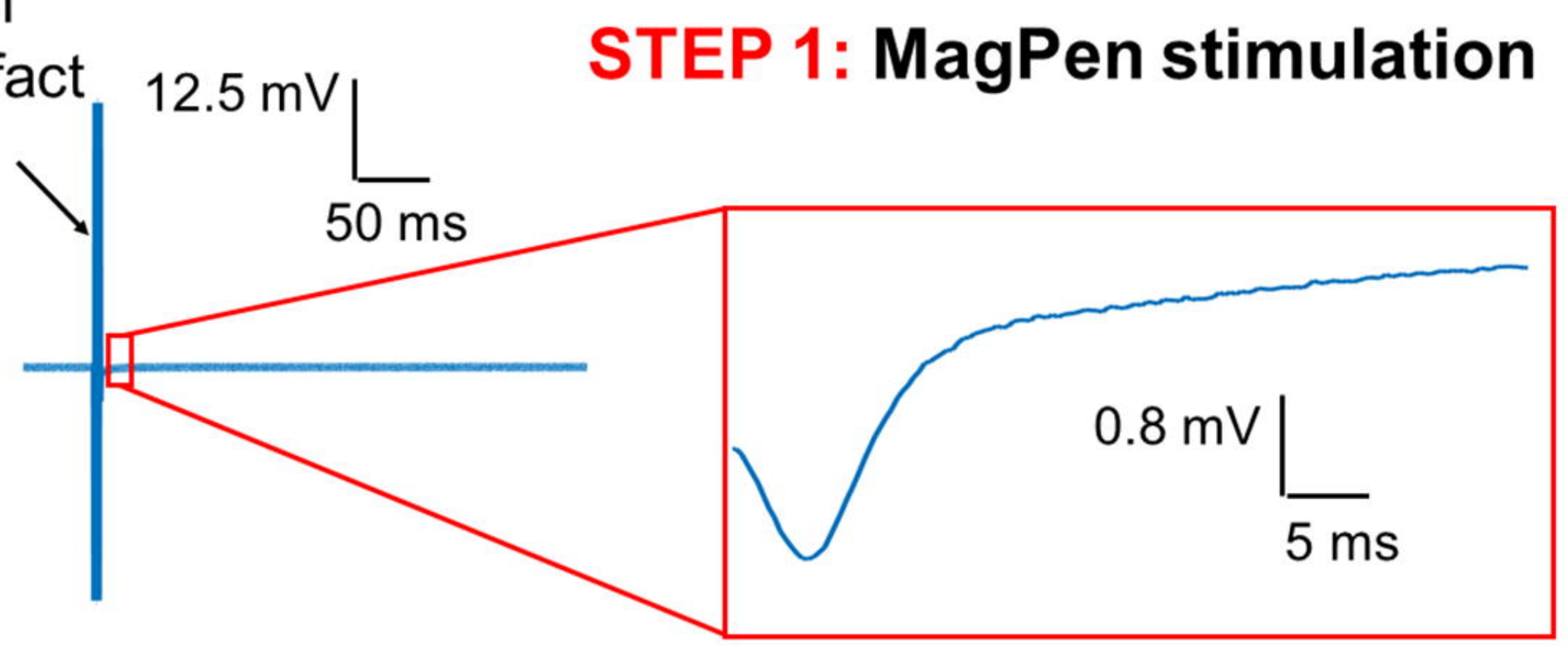

(ii)

stim

artifact

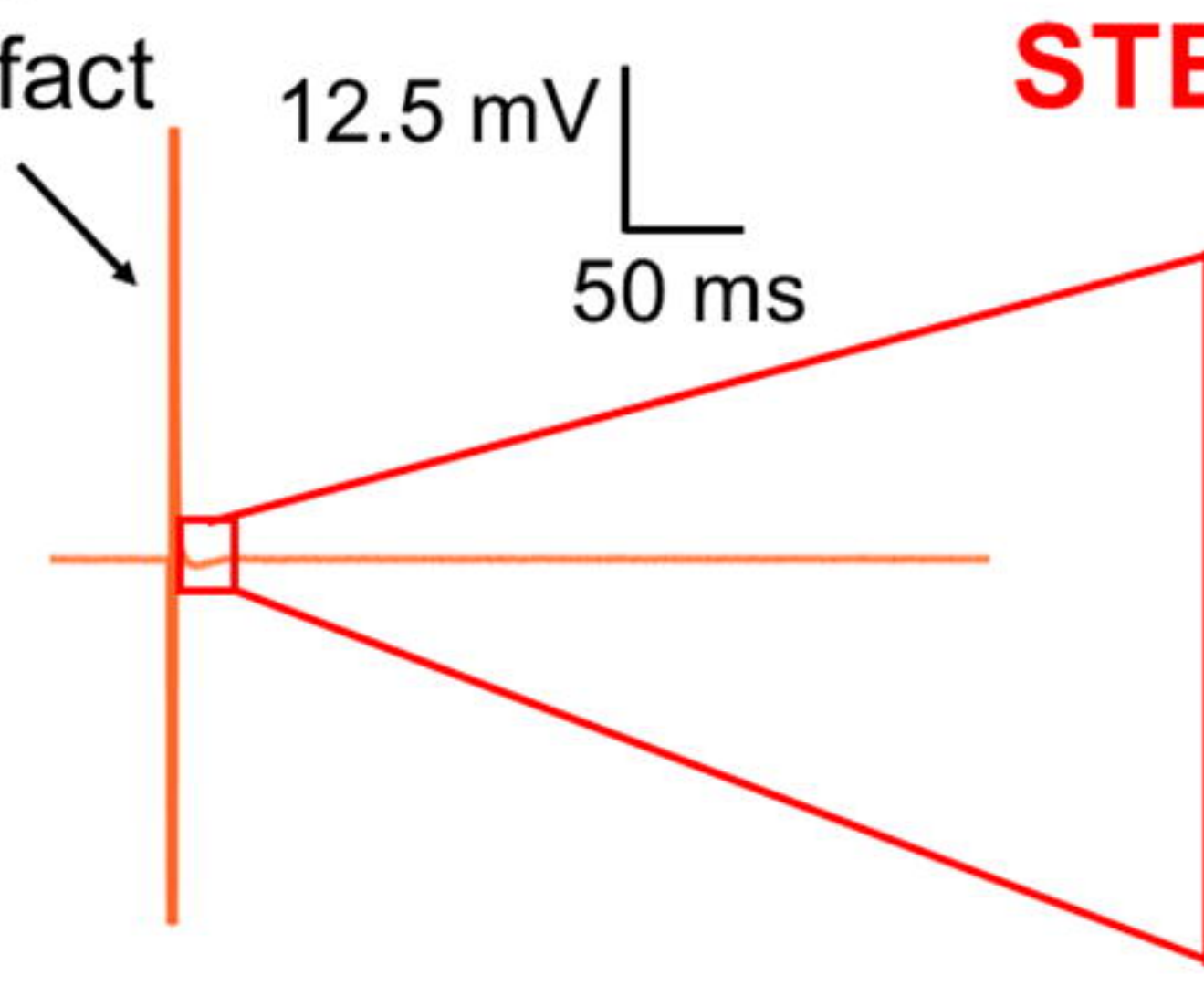

STEP 2: Apply TTX

(iii)

stim

artifact

$12.5 \mathrm{mV}$

STEP 3: TTX washout $50 \mathrm{~ms}$

(b)

- STEP 1: MagPen stimulation

- STEP 2: Apply TTX (35-40 mins)

$12.5 \mathrm{mV}$

- STEP 3: TTX washout (10 mins)

(i)

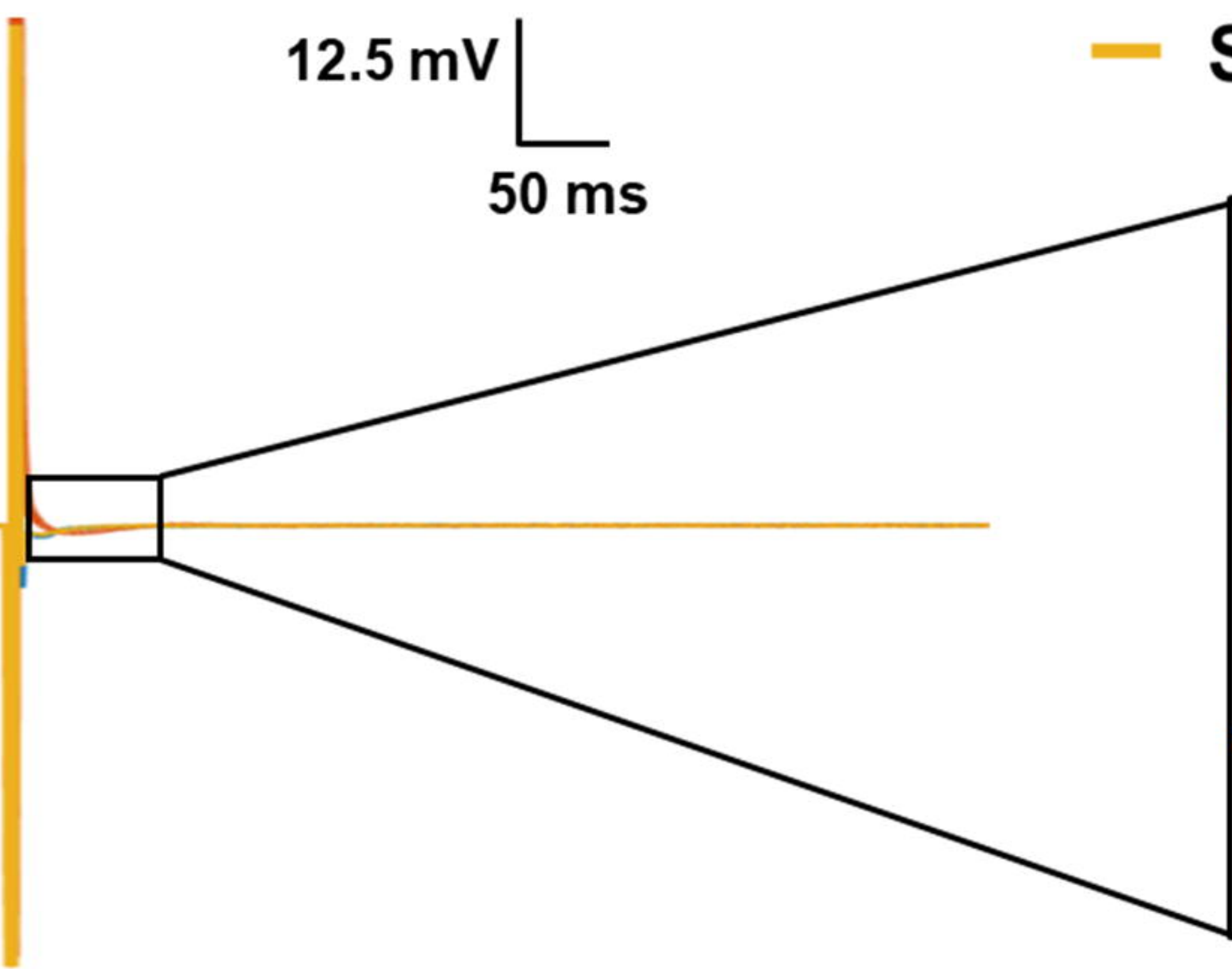

(ii)

$$
0.8 \mathrm{mv} \underset{5 \mathrm{~ms}}{\longleftarrow}
$$

